\title{
An interactive boundary layer modelling methodology for aerodynamic flows
}

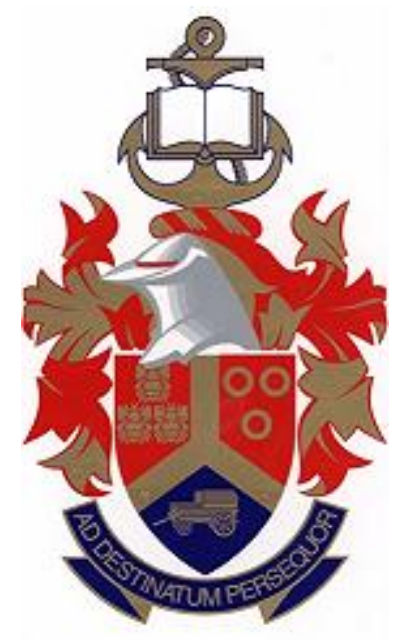

by

Lelanie Smith

Submitted in partial fulfilment of the degree

Masters of Engineering

Department of Mechanical and Aeronautical Engineering

University of Pretoria

Supervisors: Prof Dr JP Meyer, Dr OF Oxtoby and Dr AG Malan

November 2011 


\section{ABSTRACT}

An interactive boundary layer modelling methodology for aerodynamic flows

Author:

L. Smith

Supervisor:

Prof J.P. Meyer

Co-supervisors:

Dr O.F. Oxtoby

Dr A.G. Malan

Department:

Mechanical and Aeronautical Engineering

Degree:

Masters of Engineering (Aeronautical Engineering)

Computational fluid dynamics (CFD) simulation is a computational tool for exploring flow applications in science and technology. Of central importance in many flow scenarios is the accurate modelling of the boundary layer phenomenon. This is particularly true in the aerospace industry, where it is central to the prediction of drag.

Modern CFD codes as applied to modelling aerodynamic flows have to be fast and efficient in order to model complex realistic geometries. When considering viscous flows, the boundary layer typically requires the largest part of computational resources. To simulate boundary layer flow with most current CFD codes, requires extremely fine mesh spacing normal to the wall and is consequently computationally very expensive. Boundary layer modelling approaches offer considerable computational cost savings.

One boundary layer method which proved to be very accurate is the two-integral method of Drela (1985). Coupling the boundary layer solution to inviscid external flow, however, is a challenge due to the Goldstein singularity, which occurs as separation is approached.

This research proposed to develop a new method to couple Drela's two-integral equations to a generic outer flow solver in an iterative fashion. The study introduced an auxiliary equation, which was solved along with the displacement thickness to overcome the Goldstein singularity without the need to solve the entire flow domain simultaneously. In this work, the incompressible Navier-Stokes equations were used for the outer flow.

In the majority of previous studies, the boundary layer thickness was simulated using a wall transpiration boundary condition at the interface between viscous and inviscid flows. This boundary condition was inherently non-physical since it added extra mass into the system to simulate the effects of the boundary layer. Here, this drawback was circumvented by the use of a mesh movement algorithm to shift the surface of the body outward without regridding the entire mesh. This replaced the transpiration boundary condition. 
The results obtained show that accurate modelling is possible for laminar incompressible flow. The predicted solutions obtained compare well with similarity solutions in the case of flat and inclined plates, and with the results of a NACA0012 airfoil produced by the validated XFOIL code (Drela and Youngren, 2001).

Keywords: boundary layer, two-integral method, coupling, auxiliary velocity, displacement thickness, mesh movement algorithm. 


\section{ACKNOWLEDGEMENTS}

I wish to express my sincere gratitude to my supervisor, Dr OF Oxtoby, for his continual support and insight, his guidance and unlimited patience, his friendship and commitment to me and my project. It was an honour and privilege working with him and being exposed to his infinite source of knowledge in the field of computational fluid dynamics.

I would also like to thank my co-supervisor Dr AG Malan, for his encouragement and guidance during challenging phases of my project. Also for the use of Elemental, which he has crafted and developed brilliantly.

I wish to express my deepest respect and gratitude to my other co-supervisor and academic mentor Prof JP Meyer, for his continual support and advice on all levels of my academic career. It is an absolute privilege to work with him and be associated with the Department of Mechanical and Aeronautical Engineering which flourishes under his knowledgeable guidance.

I would like to acknowledge the financial support of the University of Pretoria, NRF, TESP, SOLAR Hub with the Stellenbosch University, EEDSM Hub and the CSIR.

Finally, I would like to thank my family for their support and encouragement. My dear friends who inspire and motivate me to greatness. My fellow Master students for all the entertainment and unconditional support. Lindi Maritz, Jeanette Schlebusch and Liesl Gouws for their technical assistance. Zanete Osner for her continual supportive and loving care during challenging phases of my project. My sincere gratitude to Carley and Alan Louw, and all the students and fellow teachers at Yoga Connection for being a source of strength, inspiration, energy and hours of guidance on a personal level.

"There is no such thing as a simple act of compassion or an inconsequential act of service. Everything we do for another person has infinite consequences"

- Caroline Myss 


\section{TABLE OF CONTENTS}

ABSTRACT. i

ACKNOWLEDGEMENTS .iii

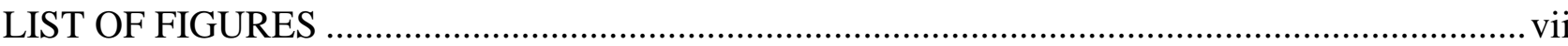

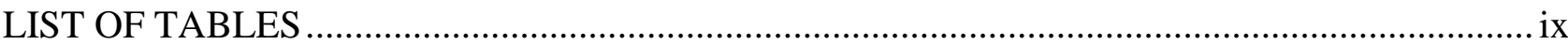

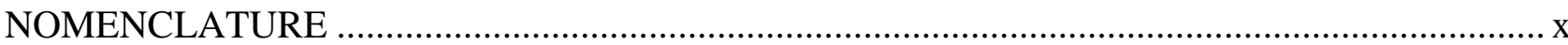

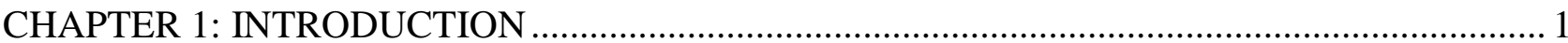

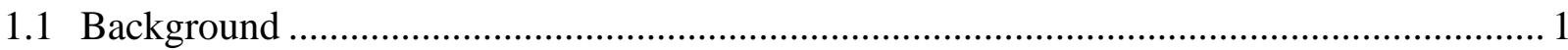

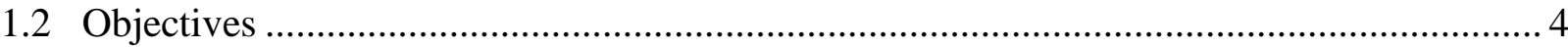

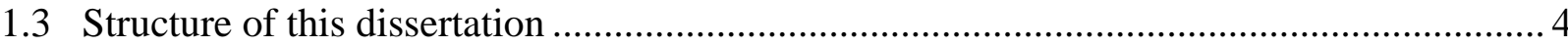

CHAPTER 2: BOUDARY LAYER MODELLING METHODS …......................................... 6

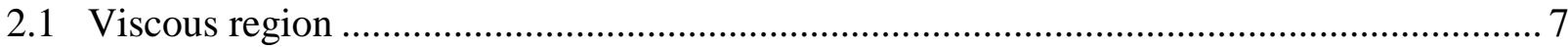

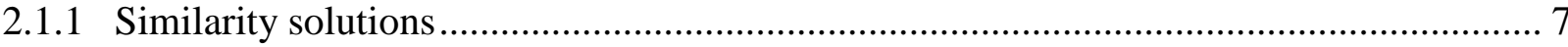

2.1.2 Numerical solutions of the boundary layer equations .............................................. 9

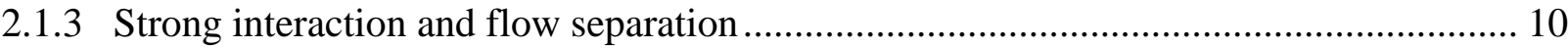

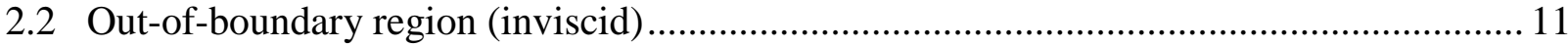

2.2.1 Summary of numerical approaches for inviscid flow equations .................................. 12

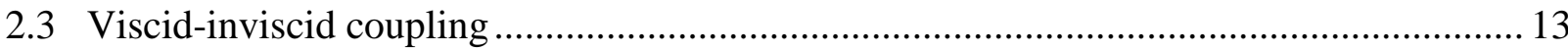

2.3.1 Interactive boundary layer modelling techniques ................................................. 14

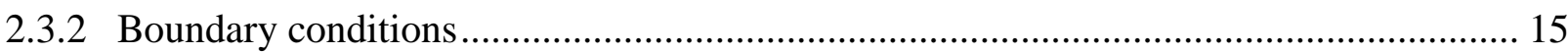

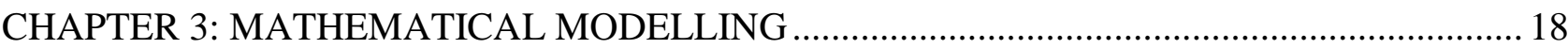

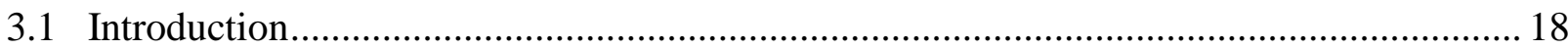

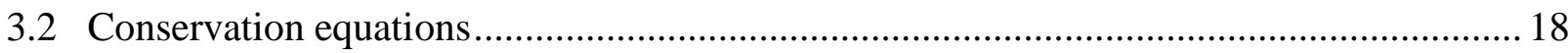

3.3 Governing equations for inviscid (out-of-boundary layer) flows ................................... 19

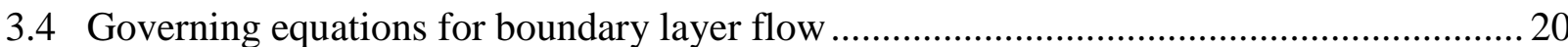

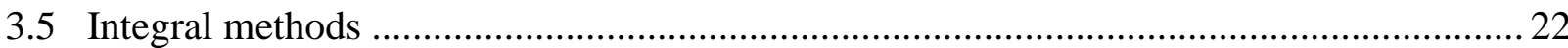




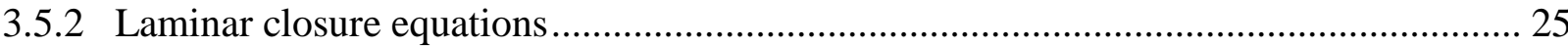

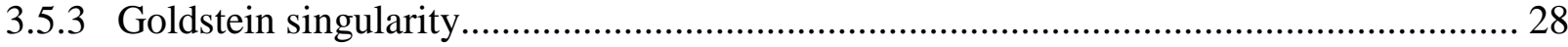

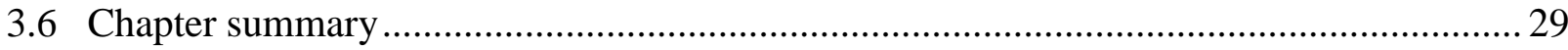

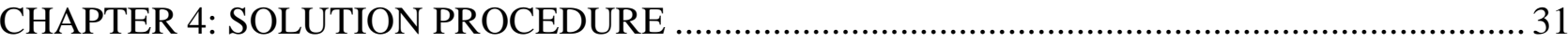

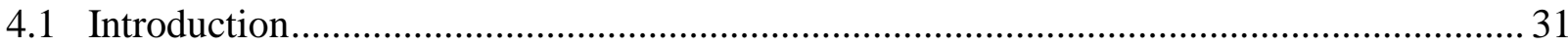

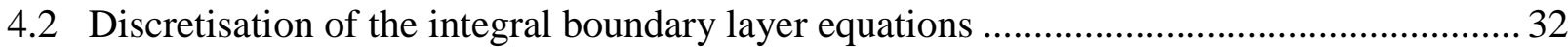

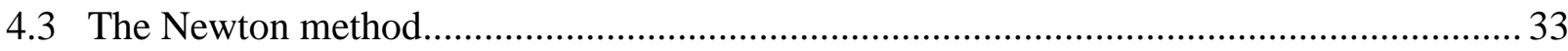

4.3.1 The algorithm for Newton's method for non-linear systems …................................. 34

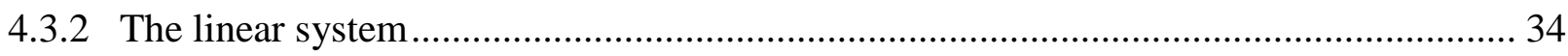

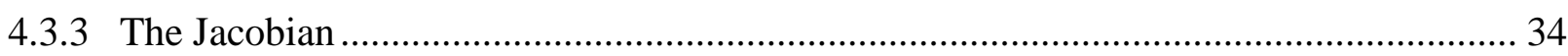

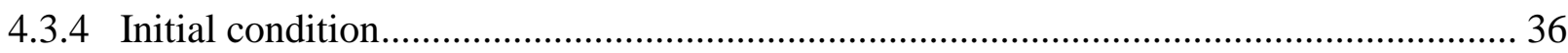

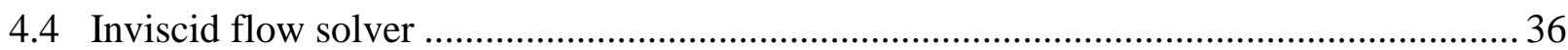

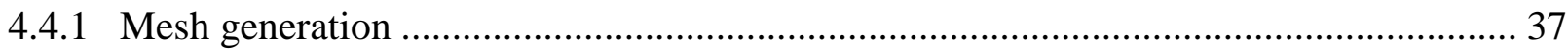

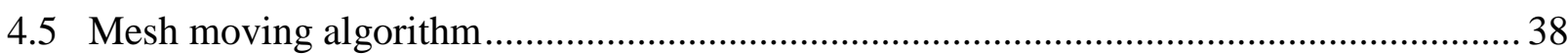

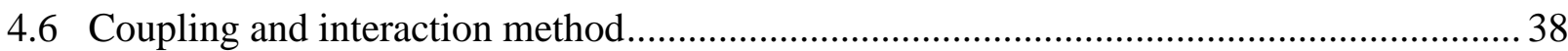

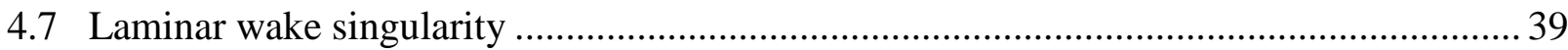

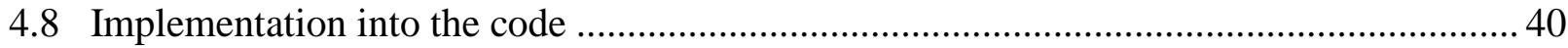

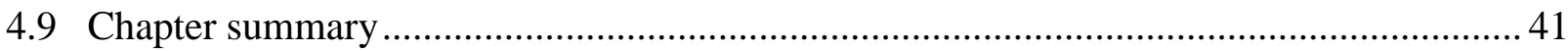

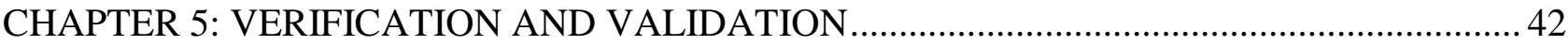

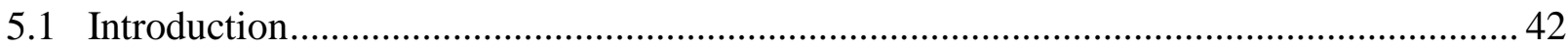

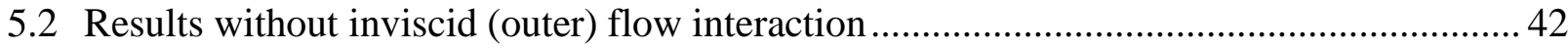

5.2.1 Steady incompressible laminar flow over a flat plate............................................... 42

5.2.2 Incompressible laminar flow over an inclined plate .............................................. 44

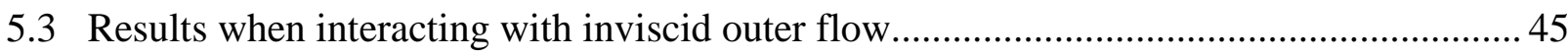

5.3.1 Incompressible laminar flow over a flat plate ....................................................... 45

5.3.2 Incompressible laminar flow over an inclined plate............................................ 46 
5.3.3 Incompressible laminar flow over a NACA0012 airfoil .............................................. 49

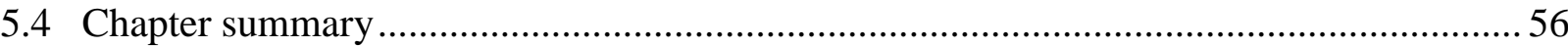

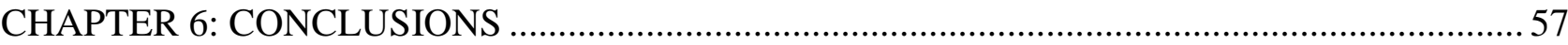

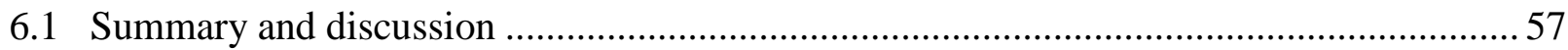

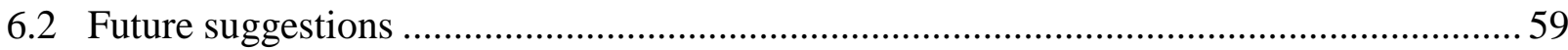

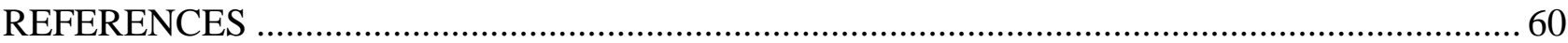




\section{LIST OF FIGURES}

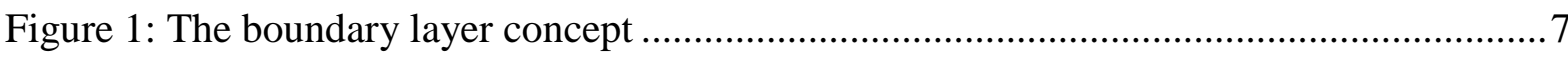

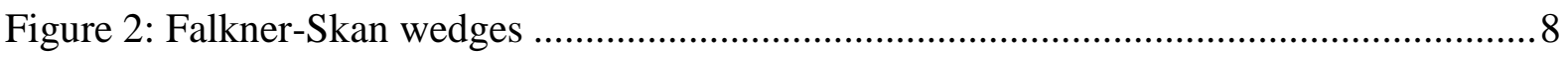

Figure 3: Equivalent fictitious flow, (a) "solid" displacement, (b) transpiration velocity

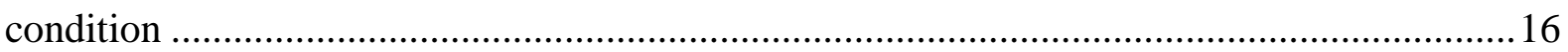

Figure 4: Laminar closure relationship for the energy thickness shape parameter.................26

Figure 5: Laminar closure relationship for the skin friction coefficient ................................2 27

Figure 6: Laminar closure relationship for the dissipation coefficient ................................27

Figure 7: Comparison between the Blasius solution and the numerical solution $\left(\Delta \boldsymbol{x}_{1}=\mathbf{0 . 0 5}\right) 43$

Figure 8: Convergence rate for the Blasius solution using different grid spacings with the dashed line depicting formal second-order accuracy..................................................... 44

Figure 9: Displacement thicknesses of flow over an inclined plate for various angles of attack

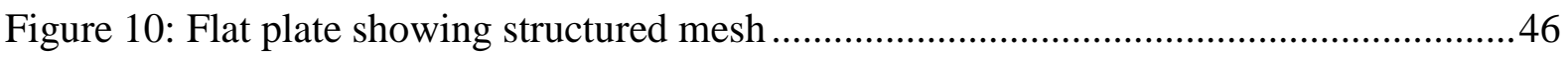

Figure 11: Close-up of mesh movement for flat plate flow in Figure 11, magnified 200 times

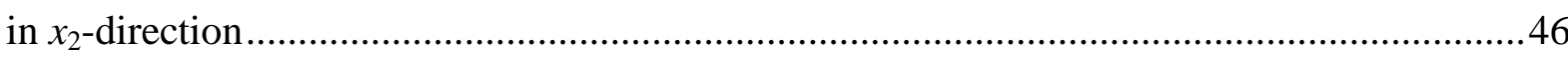

Figure 12: Comparison between the two-integral solution and the Falkner-Skan similarity solution.

Figure 13: Pressure contours and unstructured mesh $(\beta=0.4)$. Pressure values are in $\mathrm{Pa} \ldots \ldots . . .48$

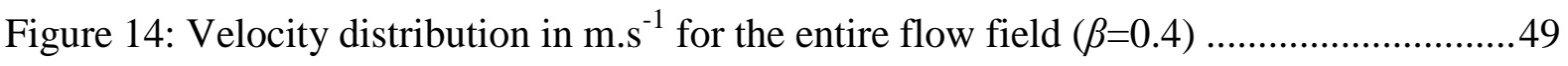

Figure 15: Mesh movement on the $\beta=0.4$ slope mesh, magnified 30 times ......................... 49

Figure 16: Comparison of the displacement thickness at different stages of convergence for

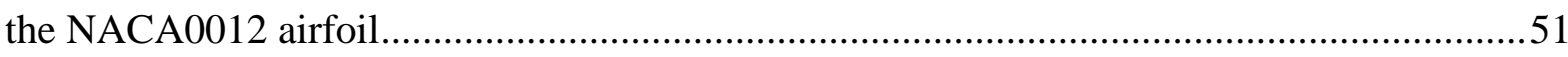

Figure 17: Comparison of velocity distribution for the NACA0012 airfoil ..........................51

Figure 18: Shape factor at different stages of convergence ….........................................52

Figure 19: Skin friction coefficient at different stages of convergence ................................52

Figure 20: Comparison between the velocity specified and the velocity obtained from the boundary layer solution, after first iteration (top), intermediate (middle) and converged (bottom)...... 
Figure 21: Mesh and velocity contours around a NACA0012 airfoil. Viscous-inviscid flow (top) and inviscid flow (bottom). Velocity distribution in $\mathrm{m}^{-1} \mathrm{~s}^{-1}$

Figure 22: Magnification of the flow across the leading edge of a NACA0012 airfoil.

Velocity distribution in $\mathrm{m} . \mathrm{s}^{-1}$

Figure 23: Mesh movement around the NACA0012 airfoil .56 


\section{LIST OF TABLES}

Table 1: Comparison between different mesh sizes for a flat plate .43

Table 2: Comparison between different mesh sizes for angled plates................................. 44

Table 3: Numerical statistics surrounding convergence as shown in Figure 12....................48 


\section{NOMENCLATURE}

$c$

$c$

$C_{D}$

$C_{f}$

$d$

$f$

$h$

$h$

H

$H^{*}$

$H^{* *}$

$J$

$k$

$L$

$M$

$\underline{n}$

$p$

$\mathrm{Re}$

$t$

$u$

$U$

V

$\dot{V}$

$x$

Artificial compressibility pseudo-acoustic velocity $\mathrm{m} \cdot \mathrm{s}^{-1}$

Chord length

$\mathrm{m}$

Dissipation coefficient

Skin friction coefficient

Original grid position

Force component

$\mathrm{N}$

Enthalpy

J.kg-1

Height

$\mathrm{m}$

Shape factor

Energy thickness shape factor

Density thickness

Jacobian

Thermal conductivity

$\mathrm{W} \cdot \mathrm{m}^{-1} \cdot \mathrm{K}^{-1}$

Length

m

Mach number

unit vector normal to the boundary

Pressure

N.m - $^{2}$

Reynolds number

Time

S

Velocity component

$\mathrm{m} \cdot \mathrm{s}^{-1}$

Free-stream velocity

$\mathrm{m} \cdot \mathrm{s}^{-1}$

Volume

$\mathrm{m}^{3}$

Volume flow rate

$\mathrm{m}^{3} \cdot \mathrm{s}^{-1}$

Length

m

Greek letters

$\alpha$

Under-relaxation parameter

$\beta$

Wedge angle

radians 


$\begin{array}{lll}\delta & \text { Boundary layer thickness } & \mathrm{m} \\ \delta^{*} & \text { Boundary layer displacement thickness } & \mathrm{m} \\ \delta^{* *} & \text { Boundary layer thickness } & \mathrm{m} \\ \delta_{i j} & \text { Kronecker delta } & \\ \delta & \text { Displacement grid position } & \\ \Delta & \text { Difference } & \\ \eta & \text { Similarity coordinate } & \\ \theta & \text { Momentum thickness } & \mathrm{m} \\ \mu & \text { Dynamic viscosity } & \mathrm{kg} \cdot \mathrm{m}^{-1} \cdot \mathrm{s} \\ \rho & \text { Density } & \mathrm{kg} \cdot \mathrm{m}^{-3} \\ \tau & \text { Shear stress } & \mathrm{N} \cdot \mathrm{m}^{-2} \\ v & \text { Kinematic viscosity } & \mathrm{m}{ }^{2} \cdot \mathrm{s}^{-1}\end{array}$

Subscripts

$\begin{array}{ll}1,2 & \text { Variables in the } x \text {-and } y \text {-directions, respectively } \\ b & \text { Blowing } \\ e & \text { Edge } \\ i, j, k & \text { Nodal point indexing in the } x \text {-, } y \text { - and } z \text {-directions, respectively } \\ k & \text { Kinematic } \\ m & \text { Mid-point between nodes } i \text { and } j \\ w & \text { Wall } \\ \theta & \text { Momentum thickness } \\ \infty & \text { Free-stream value }\end{array}$

Superscripts

$m \quad$ Exponent of the power law

$\sim \quad$ Intermediate quantity 


\section{CHAPTER 1 \\ INTRODUCTION}

\subsection{Background}

Computational fluid dynamics (CFD) is concerned with the numerical solution of equations of fluid motion as well as the interaction of fluids and solid bodies. CFD today offers software that allows the accurate simulation of transonic and turbulent flows. Modern CFD codes are an increasingly valuable design tool in engineering, as well as a substantial research tool in certain sciences. Since the 1970s, CFD codes have been used in the aerospace industry to assist in designing and optimising aircraft and jet engine configurations and performance. CFD has revolutionised airfoil design and analysis by its ability to optimise airfoil shapes to specified requirements (Versteeg and Malalasekera, 2007).

An important engineering aspect of many flow problems is the behaviour of the fluid near a solid boundary. Viscous flow moves from having completely irrotational motion away from the boundary up to the surface of the body where the velocity reaches zero, because of the noslip condition at the wall. This change occurs in a very small layer adjacent to the surface of the body, where normal and tangential forces exist not only between fluid layers but also between the fluid and the wall. The physical property of fluid responsible for these forces is viscosity. The layers in which viscous effects dominate are called boundary layers.

The boundary layer has to be resolved accurately in order to predict effects such as drag or reversed flow leading to flow separation. The boundary layer is not only important to 
determine appropriate shapes to minimise drag across a body and avoid separation but also to simulate flow through blade cascades in compressors and turbines. Drag prediction is important in the aerospace industry for economic reasons since it influences fuel burn costs (Anderson, 2007). The boundary layer solution is imperative in certain cases of separation, wakes and jet flows.

These effects are usually solved using the Navier-Stokes equations. When considering viscous flows, the boundary layer typically requires the largest part of computational resources. The reason for this is that, in boundary layer flows, gradients in velocity normal to the boundary are a factor of $\sqrt{\mathrm{Re}}$ greater than those parallel to the boundary, where Re is the Reynolds number (White, 2006). Typically, Reynolds numbers in flow over an airfoil range up to the order of $10^{6}$. This results in the need for small mesh spacing normal to the boundary. The resulting fine meshes and stability limit on time-step size mean that the boundary layer accounts for a great deal of computational cost. In addition, the need for highly stretched elements on the boundary makes the process of meshing more specialised and time-consuming. Boundary layer approaches, on the other hand, eliminate the need to resolve the boundary layer.

To describe boundary layer flow over airfoils, there are various simplifications that can be taken into account. The small thickness of the boundary layer prevalent in high Reynolds number flows at moderate angles of attack permits certain approximations within the boundary. First, the variation of the pressure normal to the wall is negligibly small. Second, the variation of velocity along the wall is much smaller than the variation of velocity normal to the wall.

Various researchers have recently achieved success in modelling boundary layers in a variety of industrially relevant scenarios. Riziotis and Voutsinas (2008), for example, improved prediction of aerodynamic performance in dynamic stall conditions of airfoils. Jie and Zhou (2008) modeled transonic flow over complex three-dimensional aircraft configurations. Sekar and Laschka (2005) determined minimum flutter speed in transonic flows and Szmelter (2001) optimised transonic wings; Florea, Hall and Cizmas (1998) modelled cases of unsteady viscous separated flow through subsonic compressors and Soize (1992) modelled unsteady compressible flow in cascade blades at positive incidences. 
In boundary layer modelling, flow is divided into two regions: an inviscid flow region, where the flow is determined from flow models such as the Euler or full potential equations, and a viscid region, where flow is governed by the boundary layer equations. Various viscidinviscid interaction techniques have been applied to find a composite solution of the boundary layer equations coupled to an approximation of the outer inviscid flow. Interactive boundary layer techniques can be extended to applications that include, multibody systems and fluid-structure interactions (Cebeci and Cousteix, 2005).

The calculation of the boundary layer equations coupled to an inviscid solution offers an attractive alternative to solving the Reynolds-averaged Navier-Stokes equations as well as to the full Navier-Stokes equations. It is computationally far less expensive since it eliminates the need to resolve the boundary layer.

However, a few difficulties are present with these methods. The main problem encountered with interactive solution techniques is the so-called 'strong interaction problem' (Wolles and Hoeijmakers, 1998). Strong interactions exist in the region of the trailing edge or where flow separation occurs, where neither the viscous nor the inviscid flow is dominant locally. It is in these cases of trailing edge and separation regions that the so-called 'Goldstein singularity' exists and where numerical interaction between the viscous and inviscid flow can fail or lack robustness (Katz and Plotkin, 2001). The interactive problem can be thoroughly analysed through the asymptotic triple-deck theory (Stewartson, 1974).

One way to overcome the Goldstein singularity is to solve the viscous and inviscid flow regions simultaneously (Drela, 1985). However, this is computationally expensive and effectively limits one to using a potential flow scheme for the inviscid flow solution. Other existing interactive methods include the semi-inverse method of Le Balleur (1983) and the quasi-simultaneous method of Veldman (1981).

Another important aspect when coupling the viscous and inviscid flow regions is that the inviscid solution needs to be informed of the boundary layer displacement. This is usually achieved by using a transpiration condition at the interface between the two flow regions (Cebeci and Cousteix, 2005). The wall transpiration condition is a non-physical condition where a fictitious velocity is induced into the boundary layer to simulate the effect of the boundary layer. 


\subsection{Objectives}

The objective of this study is to develop a method of solving boundary layer flow coupled to inviscid outer flow, which counters the difficulties described above. In order to achieve this, the researcher aims to combine the following constituents:

- an interactive solution technique to achieve computational efficiency and scaling for large problem sizes, as well as modularity of inviscid and boundary layer solvers;

- the use of a physical mass-conserving boundary condition, instead of the transpiration velocity condition;

- a coupling algorithm which circumvents the Goldstein singularity without the need for a monolithic simultaneous solution;

The algorithm developed will be used with an existing computational fluid dynamics solver to compute the influence of the boundary layer on the outer flow. The ultimate objective of this study is to present a robust solver capable of accurately modelling the boundary layer flow at a fraction of the computational cost of traditional CFD methods.

\subsection{Structure of this dissertation}

Chapter 2 gives a detailed description of how the boundary layer theory concept and the interactive boundary layer theory are modelled. This chapter also refers to the coupling between viscous and inviscid flow, with possible anomalies that should be considered.

Chapter 3 discusses the governing equations starting from the inviscid flow outside the boundary, to the different flow solutions for the boundary layer. The two-integral method of Drela (1985) is completely explained as well as the different parameters that are added to couple the viscous and inviscid flow with this method.

Chapter 4 details the complete solution procedure. The mesh movement and discretisation of the governing equations are discussed, as well as the procedure that the solver follows to calculate the boundary layer and move the mesh.

Chapter 5 gives verification and validation of the results obtained. First, the results of the approximations to the similarity solution without coupling the viscous and inviscid flow are considered, then with coupling and mesh movement. 
Chapter 6 summarises and concludes the results. Recommendations for future work and improvements are made. 


\section{CHAPTER 2 \\ BOUDARY LAYER MODELLING METHODS}

The concept of the boundary layer originated with Ludwig Prandtl in 1904, who reasoned from experimental evidence that for sufficiently large Reynolds numbers, a thin region exists near the wall where viscous effects are at least as important as inertial effects, no matter how small the viscosity of the fluid might be. Prandtl's great achievement was to show the practical importance the viscous part of the flow had on the flow solution, and which, up to that point, had been neglected to simplify the Navier-Stokes equations. Prandtl deduced that a reduced form of the governing equations could be employed under certain conditions. From this, he derived the celebrated boundary layer equations, which hold under the following two conditions:

1. The viscous layer must be thin relative to the characteristic streamwise dimension of the object immersed in the flow, $\delta / L \ll 1$, where $L$ is the characteristic length of the wall and $\delta$ is the distance away from the wall at which velocity attains its free-stream value.

2. The largest viscous term must be the same approximate magnitude as any inertia term.

Note that when the boundary layer is thinner, the smaller the viscosity, or more generally, the higher the Reynolds number. 


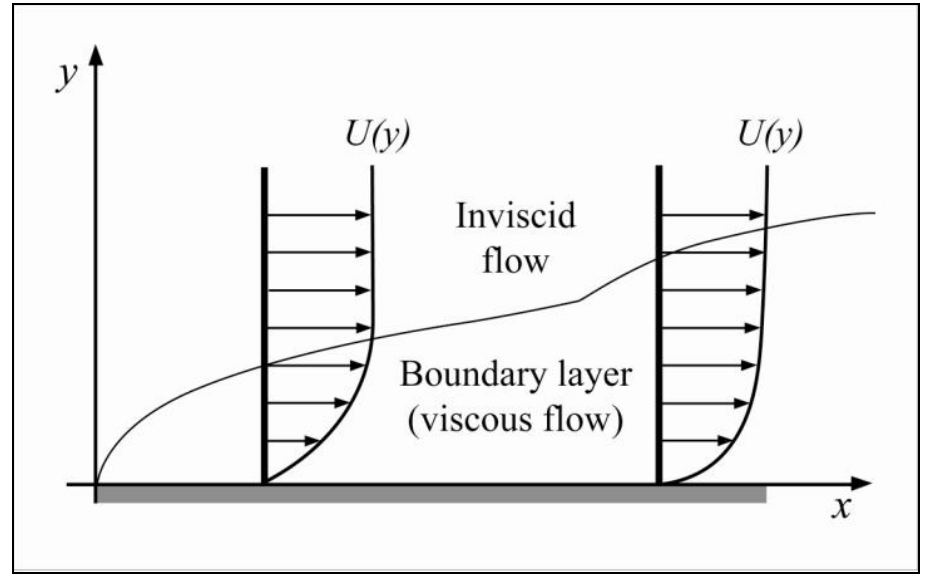

Figure 1: The boundary layer concept

The boundary layer concept supposes that fluid flow can be divided into two unequally large regions. As seen in Figure 1, in the bulk of the flow region, viscosity can be neglected; this region is called the inviscid outer flow. The second region is the very thin boundary layer at the wall where viscosity must be taken into account (Schlichting and Gersten, 2000). The methods which were used to model these two regions, as well as the coupling between them are discussed in the following section.

\subsection{Viscous region}

Prandtl showed that the Navier-Stokes equations can be simplified for application in thin boundary layers. By non-dimensionalising the equations and comparing the order of magnitude of the various terms, he showed that several terms can be neglected. In Section 3.4, these terms will be mathematically shown as well as the simplification effects on the Navier-Stokes equations to obtain the boundary layer equations. Since friction plays an important role in the boundary layer, the friction terms in the equation cannot be neglected.

The resulting two-dimensional incompressible laminar boundary layer equations can be solved either numerically or with similarity solutions. Numerical solution can either incorporate the differential method, which solves the partial differential equations or the integral method which solves the ordinary differentials that are already integrated in the $x_{2}$ direction (normal to the boundary). Further discussion of the mathematical formulation of Prandtl's reasoning is given in Section 3.4.

\subsubsection{Similarity solutions}

Blasius in 1908, was the first to use Prandtl's boundary layer equations to treat flow along a thin flat plate. Based on the premise that local velocity profiles all have the same dimensionless shape along a plate, he introduced a new independent variable, called the 
similarity variable. Using this variable he solved the continuity and momentum equations by transforming the two partial differential equations into a single ordinary differential equation.

This method was further developed by Hiemenz and Howarth (Schlichting and Gersten, 2000). Hiemenz extended the solution to include stagnation point flow. Howarth extended the Blasius series to unsymmetrical by using a power series expansion. A disadvantage of the Blasius series is that it cannot solve past the singularity that occurs at the point of separation, where the wall shear stress tends to zero. This singularity was characterised by Goldstein (1947).

Falkner and Skan (1931) extended the similarity solution by Blasius to the case where the velocity distribution of the inviscid flow is a power law, $U_{\infty}=x_{1}^{m}$. This solution illustrates both favourable and adverse pressure gradients for flow over a wedge. The Falkner-Skan family of similarity solutions provides different velocity profile solutions for various values of $\beta$ where $\beta=\frac{2 m}{m+1}$. The Falkner-Skan solution includes the Blasius solution $(\beta=m=0)$ as well as the extension of Hiemenz (Schlichting and Gersten, 2000) for the stagnation point ( $\beta$ $=m=1$ ). It also includes the point of flow separation described by $\beta=-0.199$. Figure 2 illustrates the $\beta$ angle.

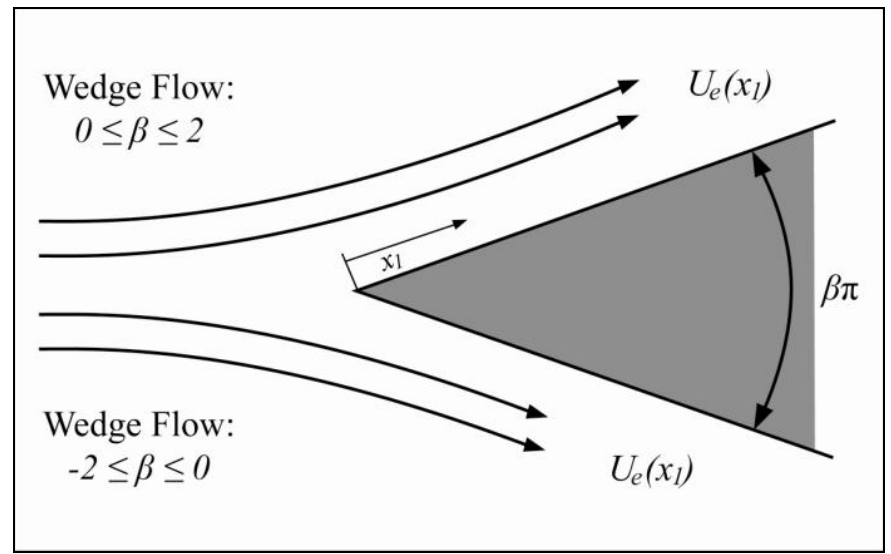

Figure 2: Falkner-Skan wedges

The different solutions of Falkner-Skan were numerically examined by Hartree and are called Hartree profiles. Hartree and Stewartson also revealed the nonuniqueness of the negative values of $m$ (Tani, 1977). Stewartson (1974) pointed that for negative $\beta$ values, $-0.199 \leq \beta \leq$ 0 , backflow occurs since there are at least two solutions for any given $\beta$. For values $\beta \leq-$ 0.199 multiple solutions exist for any given wall gradient, giving a family of separating profiles calculated by Libby and Liu (White, 2006). 
The numerical investigations of Hartree, Leigh and Terrill show that integration cannot be carried out past the separation point, further demonstrating the existence of the singularity (White, 2006). Further, all of the above work is typically limited to non-curved surfaces. In the case of airfoils, for example, numerical solution is to be sought.

\subsubsection{Numerical solutions of the boundary layer equations}

\section{Integral methods}

In practical applications, an approximate solution of the boundary layer equations is usually sufficient. Integral methods provide such an approximation. Von Karman and Pohlhausen (Katz and Plotkin, 2001) were the first to introduce the integral method. Von Karman proposed the momentum integral equation, obtained by integrating the momentum equation across the boundary layer. The remaining independent variables, therefore, are parallel to the wall. Pohlhausen applied this method to several cases using a fourth-order polynomial for the velocity distribution to develop a set of solutions including the effect of the pressure gradient inside the boundary layer.

In retarded flow regions, the approximation of Pohlhausen has less satisfactory results, for which Thwaites (Katz and Plotkin, 2001) suggested a different approximation from integrating the momentum integral equation. This method improves the original idea of Holstein and Bohlen (Katz and Plotkin, 2001), rewriting the momentum integral equation in terms of a better parameter. Thwaites looked at the entire collection of known analytical and experimental results to see if they could be fit by a set of averaged one-parameter functions.

An integral formulation of the boundary layer equations is used when coupling viscousinviscid interactive flows. This is discussed in Section 2.3. Although the boundary layer equations are simpler to solve than the complete Navier-Stokes equations, they are still nonlinear and thus pose some numerical difficulties. Special care is needed in regions where singularities occur, such as in the neighbourhood of the trailing edge and separation regions.

\section{Differential methods}

There are several numerical methods for solving the boundary layer equations in differential form. The Crank-Nicolson (Burden and Faires, 2005) and Keller box methods (Hirsch, 2001) are the most convenient ones. Of the two, the Keller box method has significant advantages over the other for two-dimensional boundary layer flows. Neither of these methods were 
specifically designed to solve the boundary but were found to have the appropriate qualities to do so accurately.

The box scheme, which is an implicit method with second-order accuracy, involves transforming the momentum equation. Instead of it being a second-order partial differential equation, it transforms into two first-order partial differential equations. This allows all the derivatives in the boundary layer equations to be approximated by simple centred differences (Keller, 1978) and two-point averages, using only values at the corners of the box. The box scheme is a flexible numerical method and can solve cases in inverse flow as well as in separation.

The use of differential methods is similar to solving the full Navier-Stokes equations in the sense that they also require small grid spacing normal to the boundary to maintain computational accuracy. In comparison with the integral methods, they are more general and accurate but computationally more expensive (Cebeci and Cousteix, 2005).

\subsubsection{Strong interaction and flow separation}

Similarity solutions (Falkner-Skan) and approximate solutions using an integral version of the boundary layer momentum equation were discussed in the previous two sections. It was briefly mentioned that Goldstein (1947) analytically showed that a singularity is present at the trailing edge and that the boundary layer could not be integrated into the wake. The source of this difficulty is the discontinuity in the boundary condition at the trailing edge where shear stress approaches zero. Goldstein also pointed out that the pressure distribution around the separation point must satisfy conditions associated with the existence of reverse flow downstream of separation.

The trailing edge is a stagnant point in the inviscid flow. The solution shows a steep decrease in surface speed as the trailing edge is approached, which corresponds to the sharp increase in pressure. The strong adverse pressure gradient in the neighbourhood of the trailing edge will lead to flow separation upstream of the edge. It appears that even in cases of flow without separation (attached flow), the boundary layer equations cannot be integrated beyond a trailing edge (Katz and Plotkin, 2001).

Goldstein (1947) derived a solution for the development of the near wake close to the trailing edge of a finite flat plate. However, this solution did not provide details in the neighbourhood of the trailing edge. Stewartson (1968) and Messiter (1970) independently derived a local 
solution that provided the bridge between the Blasius solution upstream of the trailing edge and the Goldstein near-wake solution downstream of the edge. Originally, this singularity was thought to mean that Prandtl's boundary layer equations were invalid at these points, however, Brown and Stewartson (1969) showed that a regular solution of the boundary layer equations is possible in the vicinity of the singularity, if the pressure and outer flow velocity are not prescribed in advance.

In the vicinity of these singularities, the boundary layer interacts strongly with the outer flow. The structure of the flow field changes in these cases. Instead of the usual division between inviscid flow and the boundary layer flow, there exists a three-layer hierarchical structure referred to as 'triple-deck theory' or asymptotic interaction theory (Stewartson, 1974). The boundary layer here is divided into two further layers. The triple-deck theory replaces Prandtl's theory near singular points. This analytical solution provides the displacement interaction by an asymptotic matching of flows in three layers starting at the plate.

The methods of Thwaites and Pohlhausen, mentioned earlier, tie the local profile shape to the local pressure gradient, making these one-equation integral methods unsuitable for flows with strong interaction. The two-integral method developed by Drela (1985) eliminates this direct link between profile shape and pressure gradient, improving the one-integral methods by achieving treatment of strong interactive flow. However, the equations are still singular at separation if an out-of-boundary layer velocity is specified. Interactive methods to circumvent this are discussed in Section 2.3.

\subsection{Out-of-boundary region (inviscid)}

Inviscid flow is characterised by the fact that there exist only normal pressure forces, but no tangential shear forces between the adjacent layers of flow. Inviscid flow exists away from the wall: this does not, however, mean that there is no viscosity in these regions - it merely means that the effects of viscosity are negligible. These effects are small because the velocity gradient is small and this then makes the viscous forces $\partial^{2} u / \partial x_{2}^{2}$ negligible compared with the inertial forces, which are of the order of $U_{e}^{2} / L$, where $L$ is the characteristic length of the wall. 


\subsubsection{Summary of numerical approaches for inviscid flow equations}

\section{Navier-Stokes equations}

The full system of the time-dependent Navier-Stokes equations provides the most general description of inviscid flow regions, but at the greatest computational expense.

\section{Euler equations}

The Euler equations describe the most general simplified version of the Navier-Stokes equations, where all the shear stress and heat conduction terms are neglected. Prandtl's boundary analysis shows that this is a valid approximation for flows at high Reynolds numbers outside viscous regions, which develop near solid surfaces. This is because convective effects essentially dominate flow here (Hirsch, 1995).

Euler codes are well established and can be enhanced by coupling to boundary layer solutions. Szmelter (2001) uses an inviscid-viscid coupling with Euler flow to optimise aerodynamic wings in viscous flow. It was also used by Jie and Zhou (2007) to determine transonic flow over complex three-dimensional aircraft configurations.

\section{Potential flow model}

The simplest inviscid approximation is that of the full potential model developed by Laplace and Green (Hirsch, 1995). The basic assumption of the existence of a potential inviscid flow is the condition of irrotationality, which is the condition of vanishing vorticity vector. The potential flow model can accurately predict viscous regions when coupled to the boundary layer equations, using an interactive approach. This model is useful for subsonic, low transonic and fully supersonic regimes, but outside this range, the Euler equations are advocated for the computation of inviscid flows.

Interactive approaches using the full potential flow model are those of Veldman (1981), Drela (1985), Wolles and Hoeijmakers (1998), Sekar and Laschka (2005) and Riziotis and Voutsinas (2008) to name a few. Each of these researchers used the full-potential inviscid formulation coupled to a boundary layer (viscous) formulation to evaluate various aerodynamic flow aspects, for example, viscous flow around an airfoil with separation, dynamic stall and transonic dips of airfoils. 


\subsection{Viscid-inviscid coupling}

The so-called 'viscid-inviscid interaction technique' has been applied to find an approximate solution of the Navier-Stokes equations by solving a boundary layer model coupled to an inviscid model.

\section{Weak interaction}

In design, it is common to obtain the pressure distribution about aerodynamic bodies from an inviscid flow solution. The inviscid flow solution then provides the edge velocity distribution needed as a boundary condition for solving the boundary layer equation to obtain the viscous drag on the body. The interaction between the two models is accomplished using the following procedure (Wolles and Hoeijmakers, 1998):

1. The displacement thickness is obtained by the boundary layer equations and is set as the boundary condition of the inviscid flow.

2. The displacement that the boundary layer causes induces a reaction on the outer flow, which then changes correspondingly.

3. This change then has a reaction on the boundary layer again; therefore, there is an interaction between the boundary layer and the outer flow.

4. The viscous-inviscid interaction procedure continues iteratively until the change is relatively small. In practice, however, convergence is obtained by severe under-relaxation of the changes from one iterative cycle to another (Tannehill et al., 1997).

\section{Strong interaction}

It was stated in Section 2.1 that for the limiting regions of separation and in the neighbourhood of the trailing edge, the boundary layer assumptions remain valid only if pressure and external velocity are not specified. In these regions, the flow exhibits strong interaction, and the weak interaction method described above will not converge. Instead, the boundary layer must be solved in inverse mode, meaning external velocity is not specified, by coupling the viscous and inviscid flows more tightly.

The inverse method was first developed by Catherall and Mangler (1966), who were the first to integrate the boundary layer equations through a separation point. In their method, the displacement thickness is prescribed as a boundary condition at the boundary layer edge as a function along the surface to solve the pressure field. Using this technique, they could integrate the boundary layer equations without encountering any numerical difficulties. 
One problem associated with the inverse technique is the lack of prior knowledge of the displacement thickness. This value must be obtained from solving the overall interactive method between the inviscid and boundary layer flow. Interactive approaches are therefore useful with separating flow where points of separation from the boundary occur. The next section discusses methods used to overcome this difficulty.

\subsubsection{Interactive boundary layer modelling techniques}

To avoid singularities for a viscid-inviscid interaction method, the correct treatment of the interaction rather than an adaptation of the boundary layer model itself is required. There are three basic approaches to solve the viscid-inviscid interaction problem.

In the quasi-simultaneous method of Veldman (1981), an interactive law, which models outer flow, is solved simultaneously with the boundary layer equations. In this approach, the external velocity and displacement thickness are treated as unknown quantities. While the interactive law is only a simplified model for the outer flow, the true solution can then be obtained through iterative refinement. The blowing velocity or transpiration velocity is then used to simulate the boundary layer in this region.

The quasi-simultaneous method was designed using properties of the triple-deck structure. It overcomes the Goldstein singularity by instantaneously informing the boundary layer of the effect the changes in the boundary layer have on the inviscid solver. The interactive law is then solved simultaneously with the boundary layer equations. Veldman (2009) analyses the properties this interactive law ideally has. Coenen (2001) and Cebeci and Cousteix (2005) employ the quasi-simultaneous method to model flow over two- and three-dimensional airfoil and wing flows.

Secondly, semi-inverse methods were developed by Carter (1979) and Le Balleur (1983), where coupling between inner and outer flow is achieved through a relaxation formula which successively updates the displacement thickness distribution. In this method, the boundary layer is solved in reverse, i.e. for a given displacement thickness, the velocity distribution at the edge of the boundary layer, is computed. By then comparing this computed velocity with the target distribution imposed by the inviscid flow, a relaxation formula is used to obtain new estimates for the displacement thickness (Cebeci, 1999; Lagree, 2009). 
The interactive solver of Szmelter (2001) uses the semi-inverse method of Le Balleur (1983) to improve numerical stability. Jie and Zhou (2007) also used the semi-inverse method but with a different relaxation formula by Carter (Veldman, 2009).

The fully simultaneous method of Drela and Giles (1987b), eliminates this sequential solution of equations. Instead of solving the viscid and inviscid flow using an approximate interactive law, the entire non-linear equation set is solved simultaneously as a coupled system using a global Newton method. Drela developed the XFOIL code (Drela and Youngren, 2001) to solve the interactive flow. This package has been thoroughly verified and validated for different practical airfoil problems. The results obtained in this dissertation will be compared with results obtained from the XFOIL 6.9 package.

Wolles and Hoeijmakers (1998), Sekar and Laschka (2005), Riziotis and Voutsinas (2008) are some of the authors to have used the interactive method of Drela (1985). The fully simultaneous method is more robust than the iterative methods. However, the quasisimultaneous method is simpler to implement and computationally less expensive. Also, the quasi-simultaneous method has been shown to outperform the semi-inverse method in terms of convergence speed (Lock and Williams, 1987). In this study, therefore, the methodology which is proposed to overcome the singularity is based on the quasi-simultaneous philosophy. That is, the approach taken is to solve an additional velocity equation along with the displacement thickness, which while circumventing the singularity avoids the need to solve the entire inviscid flow domain simultaneously.

\subsubsection{Boundary conditions}

The boundary condition describing the displacement effect contains the information required by the inviscid solver. This information is passed from the viscous solver to the inviscid solver by one of two boundary conditions proposed by Lighthill (1958), namely the transpiration velocity condition or the "solid" displacement surface condition. Both these conditions require the real flow in the inviscid region to be replaced by an equivalent fictitious inviscid flow to incorporate the viscous effects. It is done in such a manner that the velocity components at the edge of the boundary layer are equal in both cases (Cebeci, 1999). 


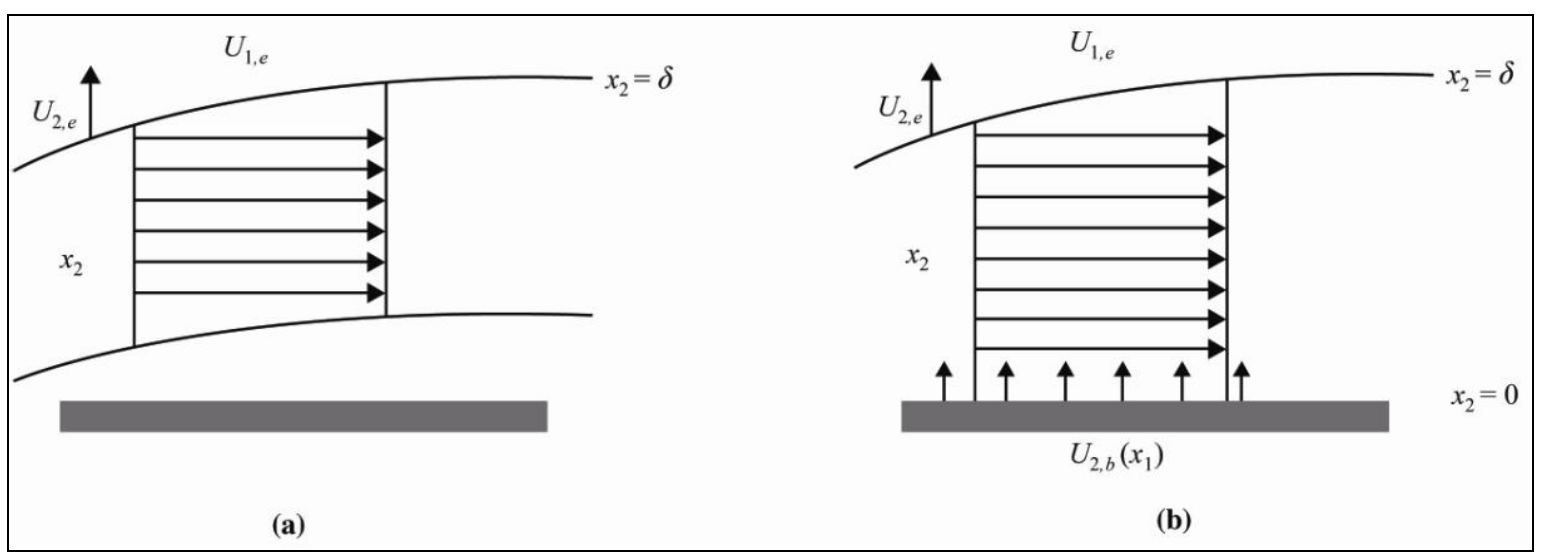

Figure 3: Equivalent fictitious flow, (a) "solid" displacement, (b) transpiration velocity condition

The transpiration condition of non-zero normal velocity at the surface, is based on the concept that the displacement surface can be formed by distributing a blowing or suction velocity on the body surface. The strength of the blowing or suction velocity $U_{2, b}$ is determined from the boundary layer solutions according to

$$
U_{2, b}=\frac{d}{d x_{1}}\left(U_{1, e} \delta^{*}\right)
$$

where $x_{1}$ is the surface distance along the body and $\delta^{*}$ is the boundary displacement thickness. The variation of $U_{2, b}$ on the body surface simulates the viscous effects in the potential flow solution (Cebeci and Cousteix, 2005). A graphical representation can be seen in Figure 3(b).

The "solid" displacement boundary condition displaces the inviscid flow by a distance equal to the displacement thickness $\delta^{*}$ formed by the boundary layer effects. The displacement distance away from the surface represents the deficiency of mass with the boundary layer (Cebeci and Cousteix, 2005). Figure 3(a) shows a graphical interpretation of this boundary condition.

Historically, the transpiration model has been preferred. One reason cited for the use of the transpiration condition is that the displacement model involves regridding the inviscid flow field, making it computationally expensive. However, moving the mesh will have the same effect without the necessity of regridding the inviscid flow field. Among the researchers who used the transpiration condition are Veldman (1981), Szmelter (2001), Jie and Zhou (2007) and Riziotis and Voutsinas (2008). 
Wolles and Hoeijmakers (1998) developed a unique way to discretise the transpiration velocity boundary condition, based on Hermite polynomial-interpolations, in their coupling of the viscid-inviscid flow scheme to overcome numerical instabilities.

The displacement method has only been used in a few cases to model separation bubbles by Gleyzes et al. (1985) and by Hafez et al. (1991) who modeled flow using a finite element interactive method. To the author's knowledge, a displacement method by moving the mesh has not been used in research until now.

The mesh movement algorithm used in this study is considered more physically realistic since it avoids the addition of spurious mass into the system by the fictitious transpiration velocity. It also avoids the complexity of calculating the effective blowing velocity necessary to simulate the boundary layer. While it does add extra complexity in the need for a mesh movement algorithm, many CFD solvers have this functionality built in anyway. 


\section{CHAPTER 3}

\section{MATHEMATICAL MODELLING}

\subsection{Introduction}

As seen in Chapter 2, finding exact solutions for the Navier-Stokes equations is generally extremely difficult. The difficulty of solving the Navier-Stokes equations numerically increases as the Reynolds number increases. However, in limiting cases of high Reynolds numbers, where non-linear inertial terms vanish in a natural way, the Navier-Stokes equations are considerably simplified. However, this limiting solution does not satisfy the no-slip boundary condition at the surface. Therefore, to satisfy this condition, viscosity has to be taken into account (Schlichting and Gersten, 2000).

Even though the boundary layer equations are considerably simpler and computationally less expensive compared with the Navier-Stokes equations, their non-linearity still makes them difficult to solve and causes methods such as super-positioning, which works well to solve inviscid, incompressible, potential flow, to fail. Therefore the boundary lay will be solved with the boundary layer equations and the out-of-boundary flow will be solved with the inviscid Euler equations.

\subsection{Conservation equations}

The fundamentals of fluid mechanics are described by the three conservation equations for mass, momentum and energy. There are certain assumptions made about the fluid in order to formulate these equations. The fluid is considered as a continuum, which means the smallest 
volume element $\delta V$ is still homogeneous. This, in turn, means that the dimensions of $\delta V$ are still very large when compared to the average distance between the molecules in the fluid. The fluid is also assumed to be a Newtonian fluid, which means that a linear relationship exists between the stress tensor and the rate of deformation tensor.

The resulting equations are:

Continuity

$$
\frac{\partial}{\partial t} \rho+\frac{\partial}{\partial x_{j}} \rho u_{j}=0
$$

Conservation of momentum (Newton's second law)

$$
\frac{\partial}{\partial t}\left(\rho u_{i}\right)+\frac{\partial}{\partial x_{j}}\left(\rho u_{i} u_{j}\right)+\frac{\partial p}{\partial x_{i}}=\rho f_{i}+\frac{\partial}{\partial x_{j}}\left[\left(\mu\left(\frac{\partial u_{i}}{\partial x_{j}}+\frac{\partial u_{j}}{\partial x_{i}}-\frac{2}{3} \frac{\partial u_{k}}{\partial x_{k}} \delta_{i j}\right)\right)\right]
$$

Conservation of energy (First law of thermodynamics)

$$
\rho \frac{D h}{D t}=\frac{D p}{D t}+\frac{\partial}{\partial x_{j}}\left(k \frac{\partial T}{\partial x_{j}}\right)+\left[\left(\mu\left(\frac{\partial u_{i}}{\partial x_{j}}+\frac{\partial u_{j}}{\partial x_{i}}-\frac{2}{3} \frac{\partial u_{k}}{\partial x_{k}} \delta_{i j}\right)\right)\right] \frac{\partial u_{i}}{\partial x_{j}}
$$

The conservation laws for the three basic flow quantities, $\rho, \rho u$ and $\rho h$ are referred to as the Navier-Stokes equations. These fundamentals may be used to calculate all flow properties and characteristics over any object. They are also the core of the boundary layer equations.

\subsection{Governing equations for inviscid (out-of-boundary layer) flows}

With the inviscid flow approximation, the continuity equation [3.1] remains unchanged. Neglecting viscosity, the Navier-Stokes equations reduce to the time-dependent Euler equations:

$$
\frac{\partial}{\partial t}\left(\rho u_{i}\right)+\frac{\partial}{\partial x_{j}}\left(\rho u_{i} u_{j}\right)+\frac{\partial p}{\partial x_{i}}=0
$$

For a steady flow with no body forces, the Euler equations are reduced to:

$$
\frac{\partial}{\partial x_{j}}\left(\rho u_{i} u_{j}\right)=-\frac{\partial p}{\partial x_{i}}
$$

These equations are compressible and can be reduced to represent steady incompressible flow when the density $\rho$ is constant. Some of the terms within the momentum equation will be neglected to simplify the boundary layer equations further for steady incompressible twodimensional laminar flow. The steady condition means that the flow is not dependent on time 
and the incompressibility conditions mean that density and viscosity are constant. Under these assumptions, the continuity equation reduces to:

$$
\frac{\partial u_{1}}{\partial x_{1}}+\frac{\partial u_{2}}{\partial x_{2}}=0
$$

Here, $u_{1}$ and $u_{2}$ are the streamwise and normal velocity components, respectively. These equations may be solved as described in Chapter 4.

\subsection{Governing equations for boundary layer flow}

The boundary layer equations are a reduced form of the Navier-Stokes equations. Two length scales are introduced near the wall, $L$, parallel to the wall, representing the length of the body and $\delta$, normal to the wall, representing the boundary layer thickness. The reduction occurs based on the premise that the length scale $\delta$ is much smaller than $L: \delta \ll L$.

It has already been stated that the higher the Reynolds number, the thinner the boundary layer, i.e. that boundary layer thickness will decrease with decrease in viscosity. The boundary layer thickness is proportional to the square root of the kinematic viscosity, $\delta \sim \sqrt{v}$. Using this relation with the free-stream velocity $U_{\infty}$ and characteristic length of the body $L$, the dimensionally correct representation is obtained:

$$
\frac{\delta}{L} \sim \frac{1}{\sqrt{\operatorname{Re}}} \text { with } \operatorname{Re}=\frac{U_{\infty} L}{v}
$$

The relationship shown in equation [3.7] shows that the larger the Reynolds number becomes, the smaller the boundary layer thickness as compared with the characteristic length of the body.

When considering flow over a flat plate (with plate normal in $\mathrm{x}_{2}$-direction), the Navier-Stokes equation in the $x_{1}$-direction is further reduced by noting that the second derivative of the velocity component in the streamwise direction, $\frac{\partial^{2} u_{2}}{\partial x_{1}^{2}}$, is negligible compared with the corresponding derivative transverse to the main flow direction, $\frac{\partial^{2} u_{1}}{\partial x_{2}^{2}}$.

The momentum equation then reduces to:

$$
u_{1} \frac{\partial u_{1}}{\partial x_{1}}+u_{2} \frac{\partial u_{1}}{\partial x_{2}}=-\frac{1}{\rho} \frac{\partial p}{\partial x_{1}}+v\left(\frac{\partial^{2} u_{1}}{\partial x_{2}^{2}}\right) \text { for the } x_{1} \text {-direction. }
$$

Typically to high Reynolds number flows, buoyancy effects within the flow do not contribute to the acceleration of the flow in the $x_{2}$-direction. The pressure gradient in the $x_{2}$-direction is 
then nearly zero, being only affected by these terms. For this reason, in the boundary layer equations, the transverse pressure gradient is negligible, following as equation [3.9] (White, 2006):

$$
\frac{\partial p}{\partial x_{2}}=0 \quad \text { for the } x_{2} \text {-direction. }
$$

Prandtl showed that pressure must be treated as a known variable in the boundary layer analysis with $p\left(x_{1}\right)$ assumed to be imposed on the boundary layer from an inviscid outer flow analysis. That is, the free--stream outside the boundary layer, $U_{e}=U_{e}\left(x_{1}\right)$, where $x_{1}$ is the coordinate parallel to the wall, is related to $p\left(x_{1}\right)$ by Bernoulli's theorem for incompressible flow (White, 2006).

Equation [3.9] states that pressure across the boundary, normal to the surface, is constant. It is for this reason that the pressure is taken to be the inviscid pressure evaluated on the surface. Application of the momentum equation [3.8] on the outer edge of the boundary is represented as

$$
U_{e}\left(x_{1}\right) \frac{\partial U_{e}\left(x_{1}\right)}{\partial x_{1}}=-\frac{1}{\rho} \frac{\partial p}{\partial x_{1}}
$$

This equation can be substituted into equation [3.8], resulting in the pressure no longer being unknown.

The following initial conditions apply to the boundary layer theory:

$$
\begin{aligned}
& u_{1}\left(0, x_{2}\right)=u_{1,0}\left(x_{2}\right) \\
& u_{2}\left(0, x_{2}\right)=u_{2,0}\left(x_{2}\right) \\
& \tau\left(0, x_{2}\right)=\tau_{0}\left(x_{2}\right)
\end{aligned}
$$

where $\tau$ denotes the shear stress in the $x_{1}$-direction: $\tau=v \frac{\partial u_{1}}{\partial x_{2}}$.

The equations are subject to the boundary conditions:

$$
\begin{array}{ll}
u_{1}\left(x_{1}, 0\right)=u_{1, w}\left(x_{1}\right) & \text { Wall motion } \\
u_{2}\left(x_{1}, 0\right)=u_{2, w}\left(x_{1}\right) & \text { Mass flux } \\
u_{1}\left(x_{1}, x_{2, e}\left(x_{1}\right)\right)=U_{e}\left(x_{1}\right) &
\end{array}
$$

Here, $u_{1, w}\left(x_{1}\right)$ simulates wall motion, $u_{2, w}\left(x_{1}\right)$ simulates a mass flux (blowing) into the boundary layer, $x_{2, e}$ denotes the 'edge' of the boundary layer and $U_{e}\left(x_{1}\right)$ is the free-stream velocity. 
This set of equations can be solved exactly using similarity solutions, however, this is only possible for simple geometries. Otherwise the equations have to be solved numerically using the integral method for more complex flow problems. The numerical scheme that will be developed for this study will, however, be tested against the similarity solutions for the flat, inclined plates and a generated XFOIL solution for a NACA0012 airfoil.

\subsection{Integral methods}

The integral solution of the boundary layer solutions is attributed to Von Karman. The momentum integral method is a second method used to approximate the boundary layer problem. Although it is an approximation solution, it does not depend on the similarity assumption and it allows for significant changes in the shape of the boundary layer velocity profile. Consequently, this method can be extended to any flow regime with complex geometries and includes effects such as transition and separation (Katz and Plotkin, 2001).

The integral solution serves many engineering applications where it is not necessary to know details of flow variables inside the boundary layer. For example, in the case of design analysis, only the wall shear stress is needed to calculate the drag force on the surface and the displacement thickness to allow for coupling with outer flow.

For a derivation of the integral momentum equation, consider Prandtl's boundary layer equations [3.7] and [3.8]. Integrating equation [3.8] with respect to $x_{2}$, from the wall, $x_{2}=0$, up to the boundary thickness, $x_{2}=\delta$, transforms the equation into an ordinary differential equation, known as the Von Karman integral momentum equation.

$$
\int_{0}^{\delta} u_{1} \frac{\partial u_{1}}{\partial x_{1}} d x_{2}+\int_{0}^{\delta} u_{2} \frac{\partial u_{1}}{\partial x_{2}} d x_{2}=-\frac{1}{\rho} \int_{0}^{\delta} \frac{\partial p}{\partial x_{1}} d x_{2}+v \int_{0}^{\delta}\left(\frac{\partial^{2} u_{1}}{\partial x_{2}^{2}}\right) d x_{2}
$$

Keeping in mind that $x_{2}=\delta$ in the boundary layer and that $\partial u_{1} / \partial x_{2}$ can be neglected as stated before to obtain equation [3.8], and using the continuity equation to replace the derivatives of $u_{2}$ with those of $u_{1}$, the equation [3.16] is reduced to:

$$
\frac{d}{d x_{1}} \int_{0}^{\delta} u_{1}^{2} d x_{2}-U_{e} \frac{d}{d x_{1}} \int_{0}^{\delta} u_{1} d x_{2}=-\frac{1}{\rho} \frac{d p}{d x_{1}} \delta-\frac{\tau_{w}}{\rho}
$$

where $\tau_{w}=\left.v \frac{\partial u_{1}}{\partial x_{2}}\right|_{x_{2}=0}$ which is the wall shear stress. 
This equation is further simplified by eliminating the pressure term $-\frac{1}{\rho} \frac{d p}{d x_{1}} \delta$ as per equation [3.10]. Then using the momentum equations outside the boundary layer and rearranging the terms, the following is obtained:

$$
\frac{d}{d x_{1}} \int_{0}^{\delta}\left(U_{e}-u_{1}\right) u_{1} d x_{2}+\frac{d U_{e}}{d x_{1}} \int_{0}^{\delta}\left(U_{e}-u_{1}\right) d x_{2}=\frac{\tau_{w}}{\rho}
$$

where the nomenclature is as defined previously.

\subsubsection{Two-equation integral boundary layer equations}

There are two very important requirements when formulating the two-equation integral boundary layer equations. First, the type of viscous formulation employed in design analysis has to have the capability of representing flow accurately within separation regions. Secondly, the laminar and turbulent formulations must be compatible. It was with these requirements in mind that the two-equation integral formulation based on dissipation closure was developed for both laminar and turbulent flows (Drela and Giles, 1987a).

Drela and Giles substituted equations for momentum and displacement thickness into the momentum integral equation [3.18] to obtain the momentum integral equation in terms of momentum and displacement thickness. This method is referred to as the 'two-integral method'. These equations can also be solved by using a variety of numerical schemes to obtain approximations of the real solution.

The momentum and displacement thicknesses $\theta$ and $\delta^{*}$, and the skin friction coefficient $C_{f}$ are defined as follows:

$$
\begin{aligned}
& \theta=\int_{0}^{\infty}\left(1-\frac{u}{U_{e}}\right) \frac{\rho u}{\rho U_{e}} d \eta \\
& \delta^{*}=\int_{0}^{\infty}\left(1-\frac{\rho u}{\rho U_{e}}\right) d \eta \\
& C_{f}=\frac{2}{\rho_{e} U_{e}^{2}} \tau_{w}
\end{aligned}
$$

where $\eta$ denotes the similarity variable $\eta=\sqrt{\operatorname{Re} / v x_{1} x_{2}}$.

Substituting these equations into equation [3.18], an ordinary differential equation is obtained, which contains two-integral quantities: momentum thickness and boundary layer 
thickness. Using the shape factor $H$, the friction coefficient $C_{f}$ and $M_{e}$ as the edge Mach number, a dimensionless version of the integral momentum equation is obtained:

$$
\frac{x_{1}}{\theta} \frac{d \theta}{d x_{1}}=\frac{x_{1}}{\theta} \frac{C_{f}}{2}-\left(2+H-M_{e}^{2}\right) \frac{x_{1}}{U_{e}} \frac{d U_{e}}{d x_{1}}
$$

where

$$
H=\frac{\delta^{*}}{\theta}
$$

If equation [3.9] is first multiplied by $u$ and then integrated, the kinetic energy integral equation results:

$$
\frac{x_{1}}{\theta^{*}} \frac{d \theta^{*}}{d x_{1}}=\frac{x_{1}}{\theta^{*}} 2 C_{D}-\left(\frac{2 \delta^{* *}}{\theta^{*}}+3-M_{e}^{2}\right) \frac{x_{1}}{U_{e}} \frac{d U_{e}}{d x_{1}}
$$

The kinetic energy and density thicknesses $\theta^{*}$ and $\delta^{* * *}$, and dissipation coefficient $C_{D}$, are defined by:

$$
\begin{aligned}
& \theta^{*}=\int_{0}^{\infty}\left(1-\left(\frac{u}{U_{e}}\right)^{2}\right) \frac{\rho u}{\rho U_{e}} d \eta \\
& \delta^{* *}=\int_{0}^{\infty}\left(1-\frac{\rho}{\rho_{e}}\right) \frac{u}{U_{e}} d \eta \\
& C_{D}=\frac{1}{\rho_{e} U_{e}^{3}} \int_{0}^{\infty} \tau \frac{\partial u}{\partial \eta} d \eta
\end{aligned}
$$

These equations contain three different shape parameters: the shape parameter $H$, the energy thickness shape parameter $H^{*}$, which eliminates the direct link between the $H$ and the local external velocity $U_{e}$, and the density thickness shape factor $H^{* *}$.

They are defined as follows:

$$
H=\frac{\delta^{*}}{\theta} \quad H^{*}=\frac{\theta^{*}}{\theta} \quad H^{* *}=\frac{\delta^{* *}}{\theta}
$$

Using these shape parameter definitions and subtracting equation [3.22] from equation [3.24], the momentum and kinetic energy equations can be written as:

$$
\begin{aligned}
& \frac{d \theta}{d x_{1}}+\left(2+H-M_{e}^{2}\right) \frac{\theta}{U_{e}} \frac{d U_{e}}{d x_{1}}=\frac{C_{f}}{2} \\
& \theta \frac{d H^{*}}{d x_{1}}+\left[2 H^{* *}+H^{*}(1-H)\right] \frac{\theta}{U_{e}} \frac{d U_{e}}{d x_{1}}=2 C_{D}-H^{*} \frac{C_{f}}{2}
\end{aligned}
$$

The two-integral equations [3.29] and [3.30] contain three unknowns, $\delta^{*}, \theta$ and $C_{f}$, and cannot be solved without additional information. The necessary information is obtained from 
an assumed velocity profile family, a function of the Falkner-Skan solution. In principle, this profile defines the entire boundary layer velocity field $U_{e}=u_{1}\left(x_{1}, x_{2}\right)$, which has the same form anywhere along the boundary layer.

Different integral boundary layer methods make different choices for the local boundary layer thickness and the shape of the local velocity profile. Examples of these methods are that of Pohlhausen and that proposed by Thwaites, however, these are both one-integral systems (Katz and Plotkin, 2001). Drela suggested a different set of closure equations when using the two-integral boundary layer method (Drela, 1985), which is discussed next.

\subsubsection{Laminar closure equations}

The integral boundary layer equations [3.29] and [3.30] are valid for both laminar and turbulent boundary layers, as well as for free wakes. The fundamental difficulty with these equations is that they contain more than two independent variables. Consequently, some assumptions about the additional unknowns are to be made to obtain a solution.

The two dependent variables are defined as momentum and displacement thickness, $\theta$ and $\delta^{*}$ . $U_{e}$ and $M_{e}$ relate to inviscid flow and therefore do not represent additional unknowns. The undefined variables that remain are $C_{f}, C_{D}, H^{*}$ and $H^{* *}$. The following functional dependencies are assumed (Drela and Giles, 1987a).

$$
\begin{aligned}
& H^{*}=H^{*}\left(H_{k}, M_{e}, \operatorname{Re}_{\theta}\right) \\
& H^{* *}=H^{* *}\left(H_{k}, M_{e}\right) \\
& C_{f}=C_{f}\left(H_{k}, M_{e}, \operatorname{Re}_{\theta}\right) \\
& C_{D}=C_{D}\left(H_{k}, M_{e}, \operatorname{Re}_{\theta}\right)
\end{aligned}
$$

$H_{k}$ is the kinematic shape parameter defined with density taken as constant across the boundary layer and solely depends on the velocity profile. $H_{k}$ was developed as an empirical expression by Whitfield in terms of the conventional shape parameter and edge Mach number as follows:

$$
H_{k} \equiv \frac{\int_{0}^{\infty}\left(1-\frac{u}{U_{e}}\right) d \eta}{\int_{0}^{\infty}\left(1-\frac{u}{U_{e}}\right) \frac{u}{U_{e}} d \eta}=\frac{H-0.29 M_{e}^{2}}{1+0.113 M_{e}^{2}}
$$


This expression assumes adiabatic flow and non-unity Prandtl number of air. It is accurate up to $M_{e}=3$ and used for both laminar and turbulent flows (Drela, 1985). This equation simplifies to $H_{k}=H$ for incompressible flow.

To define the shape factor correlations, the velocity profiles of the Falkner-Skan solution were used to carry out the numerical integration of the four length scales $[3.19,20]$ and $[3.25$, 26] to obtain analytical expressions of [3.28a-c]. This was done in order to reduce computational cost associated with integration of the length scales at each $x_{1}$ location (Whitfield, 1978). For laminar flow, Drela used a finite differencing method to solve the Falkner-Skan equations with prescribed shape parameter to obtain the following curve fits, referred to as 'laminar closure equations'.

First, the energy thickness shape parameter equations and visual interpretation of the relationship are shown in Figure 4.

$$
\begin{aligned}
& H^{*}=1.515+0.076 \frac{\left(4-H_{k}\right)^{2}}{H_{k}}, H_{k}<4 \\
& H^{*}=1.515+0.04 \frac{\left(H_{k}-4\right)^{2}}{H_{k}}, H_{k}>4
\end{aligned}
$$

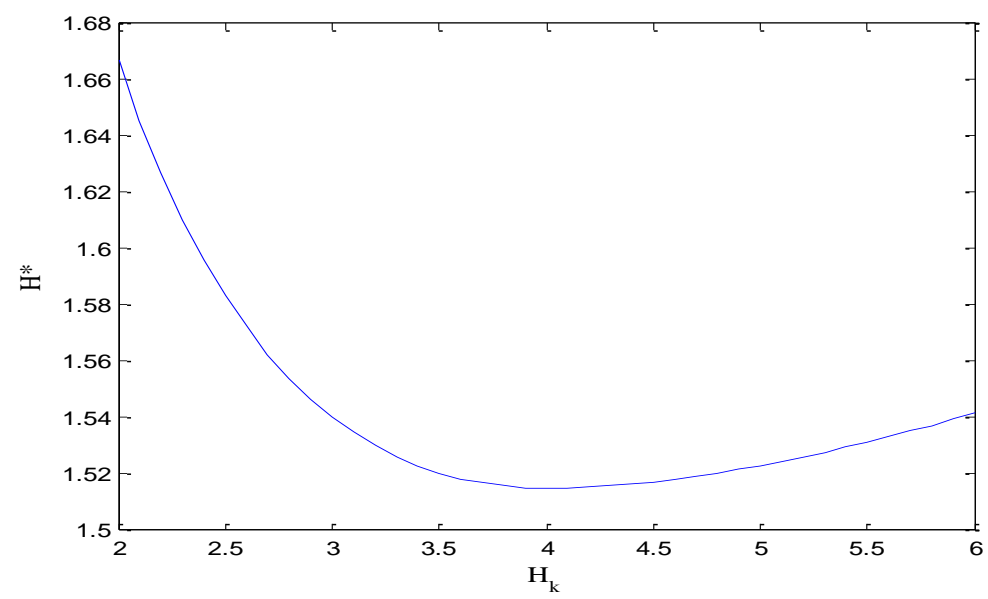

Figure 4: Laminar closure relationship for the energy thickness shape parameter

Next, the skin friction coefficient as shown is represented by Figure 5 .

$$
\begin{aligned}
& \operatorname{Re}_{\theta} \frac{C_{f}}{2}=-0.067+0.01977 \frac{\left(7.4-H_{k}\right)^{2}}{H_{k}-1}, H_{k}<7.4 \\
& \operatorname{Re}_{\theta} \frac{C_{f}}{2}=-0.067+0.022\left(1-\frac{1.4}{H_{k}-6}\right)^{2}, H_{k}>7.4
\end{aligned}
$$




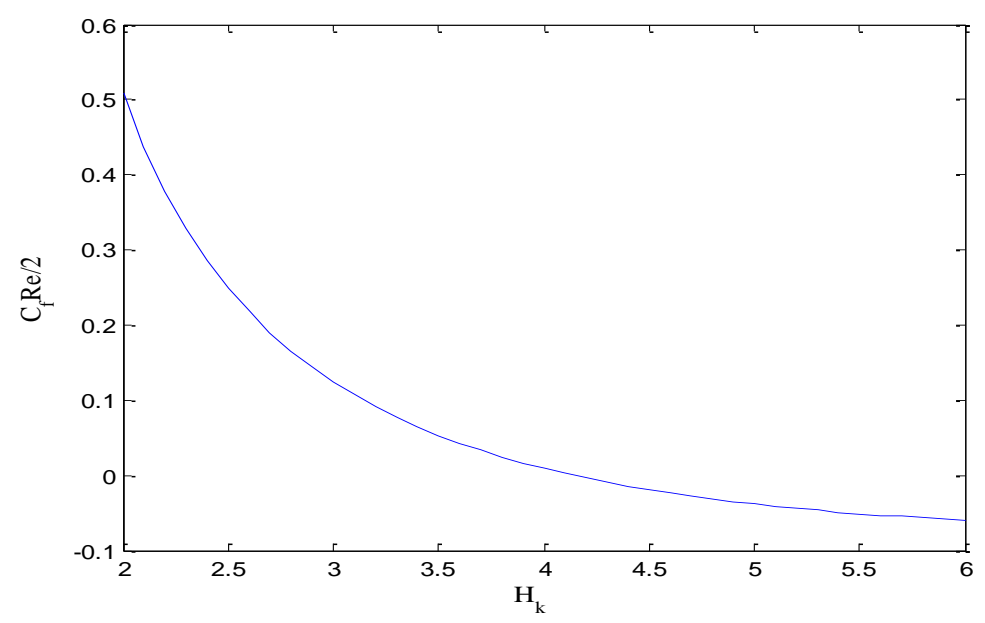

Figure 5: Laminar closure relationship for the skin friction coefficient

Last, the dissipation coefficient as shown below is given in Figure 6:

$$
\begin{aligned}
& \operatorname{Re}_{\theta} \frac{2 C_{D}}{H^{*}}=0.207+0.00205\left(4-H_{k}\right)^{5.5}, H_{k}<4 \\
& \operatorname{Re}_{\theta} \frac{2 C_{D}}{H^{*}}=0.207-0.003 \frac{\left(H_{k}-4\right)^{2}}{\left(1+0.02\left(H_{k}-4\right)^{2}\right)}, H_{k}>4
\end{aligned}
$$

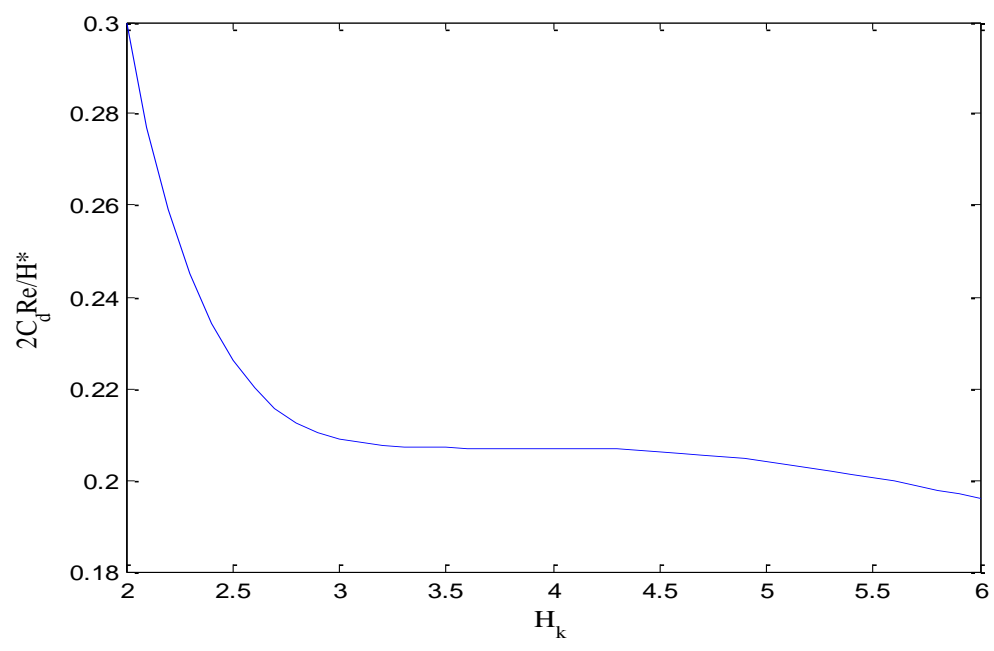

Figure 6: Laminar closure relationship for the dissipation coefficient

The density thickness $H^{* *}$ is derived by Whitfield and is defined as follows:

$$
H^{* *}=\left(\frac{0.064}{H_{k}-0.8}+0.251\right) M_{e}^{2}
$$

This shape parameter is negligible for low subsonic flows and has only a small effect on transonic flows (Drela, 1985). It is important to note the function shape of the closure 
equations [3.32 - 3.34]. They are smooth without discontinuities, which means numerically, these functions will not cause any computational instabilities.

The level of accuracy of these correlations is a consequence of the fact that almost any laminar velocity profile is very similar to the Falkner-Skan profile. This is true for measured as well as computed solutions. This makes the empirical equations [3.32 - 3.34] very accurate for all flows, especially in the incompressible case.

The integral boundary layer equations, after being simplified to two-dimensional steady incompressible laminar flow are:

$$
\begin{aligned}
& \frac{d \theta}{d x_{1}}+(2+H) \frac{\theta}{U_{e}} \frac{d U_{e}}{d x_{1}}=\frac{C_{f}}{2} \\
& \theta \frac{d H^{*}}{d x_{1}}+\left[H^{*}(1-H)\right] \frac{\theta}{U_{e}} \frac{d U_{e}}{d x_{1}}=2 C_{D}-H^{*} \frac{C_{f}}{2}
\end{aligned}
$$

For the purpose of this work, these equations will be discretised and solved by using Newton's method, after which they will be connected to a solver which solves the inviscid flow in the outer flow region.

\subsubsection{Goldstein singularity}

The laminar closure equations reach a singularity at the point where $H_{k}$ reaches 4 , which is where the function [3.32] reaches a minimum (Figure 4). This is referred to as the 'Goldstein singularity' at a boundary layer separation point. The vanishing derivative of $H^{*}$ causes a singularity in equation [3.33], which can only be avoided if $U_{e}$ adjusts to cause the rest of the equation to tend to zero as well. Therefore, any boundary layer method with a prescribed $U_{e}$ that reaches separation will fail at this point.

This problem can be circumvented by solving the inviscid flow and boundary layer simultaneously. Alternatively, elimination of this problem can be accomplished by allowing the boundary layer to modify the inviscid flow solver it is interacting with by modifying $U_{e}$ via the displacement thickness. This process creates a negative feedback effect, which can eliminate the singularity. 
This can be achieved by assuming the boundary layer is growing on the wall of a twodimensional channel, which has a fixed specified total volume flow rate $\dot{V}$ and a varying specific height $h\left(x_{1}\right)$ (Drela, 2010).

The velocity can be written as

$$
U_{e}\left(x_{1}\right)=\dot{V} /\left(h\left(x_{1}\right)-\delta^{*}\left(x_{1}\right)\right),
$$

allowing the derivation of a differential equation for $U_{e}$ :

$$
\frac{d U_{e}}{d x_{1}}=\left(\frac{d \delta^{*}}{d x_{1}}-\frac{d h}{d x_{1}}\right) U_{e} /\left(h-\delta^{*}\right) .
$$

Here, $h\left(x_{1}\right)$, the specific channel height, is calculated from the specified velocity by

$$
h\left(x_{1}\right)=\frac{\dot{V}}{U_{e, s p e c}\left(x_{1}\right)}+\delta_{\text {estimate }}^{*}\left(x_{1}\right)
$$

Where $\delta_{\text {estimate }}^{*}\left(x_{1}\right)$ is an estimated displacement thickness and $U_{e, s p e c}$ is the velocity obtained from the inviscid solution.

It is unavoidable that the final $U_{e}$ will differ slightly from $U_{e, s p e c}$, although setting $\delta_{\text {estimate }}^{*}\left(x_{1}\right)$ to the Blasius solution velocity distribution rather than zero can decrease this difference. Also, although $\dot{V}$ is an arbitrary value, it plays a role in the accuracy versus stability tradeoff. The greater the value of $\dot{V}$, the closer $U_{e}$ will be to $U_{e, s p e c}$, but if it is set too high, the Goldstein singularity is approached once again.

Equation [3.39] becomes an additional equation to solve with equations [3.36] and [3.37]. This allows the simultaneous solution of displacement thickness and velocity, circumventing the singularity, while avoiding the need to solve the entire inviscid flow domain simultaneously.

\subsection{Chapter summary}

In this chapter, the general governing equations of fluid flow were presented. These equations were used to derive the two-integral method of Drela (1985). Closure equations were derived numerically from the similarity solutions of the Falkner-Skan equations and were discussed. The velocity equations to overcome the Goldstein singularity that exists at the trailing edge 
were also presented here. The solution procedure of the two-integral method will be discussed in Chapter 4. 


\section{CHAPTER 4 \\ SOLUTION PROCEDURE}

\subsection{Introduction}

The inviscid flow Euler and continuity equations [3.5 - 3.6] are solved in a two-dimensional domain, while the boundary layer equations are solved on a one-dimensional grid. The boundary layer equations involve coupled non-linear ordinary differential equations, which were set up in Chapter 3 as:

$$
\begin{gathered}
\frac{d \theta}{d x_{1}}=\frac{C_{f}}{2}-(2+H) \frac{\theta}{U_{e}} \frac{d U_{e}}{d x_{1}} \\
\theta \frac{d H^{*}}{d x_{1}}=2 C_{D}-H^{*} \frac{C_{f}}{2}-\left[H^{*}(1-H)\right] \frac{\theta}{U_{e}} \frac{d U_{e}}{d x_{1}} \\
\frac{d U_{e}}{d x_{1}}=\left(\frac{d \delta^{*}}{d x_{1}}-\frac{d h}{d x_{1}}\right) U_{e} /\left(h-\delta^{*}\right)
\end{gathered}
$$

where $h$ is obtained as per [3.40].

Numerically solving the boundary layer equations as discussed in Chapter 3 is done as follows:

- The differencing method is selected as Crank-Nicolson for its second-order accuracy and numerical stability.

- A solution method for the system of ordinary differential equations is selected to iteratively solve the set of non-linear equations (boundary layer equations). The 
problem is treated as an initial value problem, solved point by point using Newton's method.

- The boundary layer solution is coupled with the inviscid flow solver using a mesh movement algorithm. An under-relaxation parameter is used to ensure convergence.

- The Goldstein singularity, which exists when $H \rightarrow 4$, is overcome by solving an additional velocity equation suggested by Drela (2010).

- The laminar wake, which is beyond the scope of this study, is treated with an approximation function to simulate this region.

From equations [4.1 - 4.3], it is evident that the boundary layer equations are only solved in the $x_{1}$-direction. This is because, to obtain the Von Karman integral equations, the equations are integrated in the $x_{2}$-direction, leaving them dependent on $x_{1}$ only.

For the inviscid flow, a two-dimensional domain of $x_{1}$ and $x_{2}$ is used to solve the complete inviscid flow field. The one-dimensional mesh obtained to solve the boundary layer equations is determined by the intersection between the inviscid flow mesh and the wall.

\subsection{Discretisation of the integral boundary layer equations}

The approximate solution for the boundary equations here is obtained by solving them as an initial value problem rather than simultaneously solving the whole complex system in one step. Starting at a given initial value, approximations are obtained for the displacement thickness $\delta^{*}$ and momentum thickness $\theta$. The initial starting value is obtained from the similarity solution of Blasius for a flat plate.

There are two differential equations for the laminar incompressible flow that have to be discretised, namely the momentum equation [4.1] and the shape parameter equation [4.2]. A third velocity equation [4.3] is also discretised. The independent variables are $\delta^{*}, \theta$ and $U_{e}$.

Stepping through space by an amount $\Delta x_{1}$, the other points in the domain are solved using the implicit Crank-Nicolson differencing method, with a non-linear equation solver such as Newton's method. The Crank-Nicolson scheme is unconditionally stable while being secondorder accurate, therefore, there are no restriction on the step size and hence no restriction on the mesh. The discretised form of the momentum equation is: 


$$
f_{1} \equiv \frac{\theta_{i}-\theta_{i-1}}{\Delta x_{1, i}}+\left(2+H_{m}\right) \frac{\theta_{m}}{U_{e, m}} \frac{U_{e, i}-U_{e, i-1}}{\Delta x_{1, i}}-\frac{C_{f, m}}{2}=0,
$$

where $C_{f}=C_{f}\left(H_{k}, R e\right)$ as given by [3.33] and the subscript $m$ refers to a value halfway between $i$ and $i-1$, for example, $\theta_{m}=1 / 2 \theta_{i}+1 / 2 \theta_{i-1}$.

The shape parameter equation is discretised as:

$$
f_{2} \equiv \theta_{m} \frac{H_{i}^{*}-H_{i-1}^{*}}{\Delta x_{1, i}}-2 C_{D, m}+H_{m}^{*} \frac{C_{f, m}}{2}+H_{m}^{*}\left(1-H_{m}\right) \frac{\theta_{m}}{U_{e, m}} \frac{U_{e, i}-U_{e, i-1}}{\Delta x_{1, i}}=0,
$$

where $C_{f}=C_{f}\left(H_{k}, \mathrm{Re}\right)$ as given by [3.33], $C_{D}=C_{D}\left(H_{k}, \mathrm{Re}\right)$ as given by [3.34]. $H_{k}=H=\delta^{*} / \theta$ and $H^{* *}=O$ since it is incompressible attached flow.

Finally, the velocity equation, included to overcome the Goldstein singularity near the trailing edge, is discretised as:

$$
f_{3} \equiv \frac{U_{e, m}}{\Delta x_{1, i}\left(h_{m}-\delta_{m}^{*}\right)}\left(\delta_{i}^{*}-\delta_{i-1}^{*}-h_{i}+h_{i-1}\right)-\frac{U_{e, i}-U_{e, i-1}}{\Delta x_{1, i}}=0,
$$

where $h$ is the channel height as defined in equation [3.40].

\subsection{The Newton method}

The Newton method is a well-established algorithm for solving non-linear scalar or vector equations. It is fast to converge, if the initial approximation is close to the solution. Equations [4.4 - 4.6] are solved as an initial value problem using a point-by-point local Newton method, contrary to the method by Drela (1985), which uses a global Newton method to solve simultaneously for all the points as well as the potential inviscid flow. Solving the equations in this manner is much less computationally expensive since it does not solve the whole monolithic system with each iteration. With the Newton method in this work, the exact Jacobian matrix is determined at each iteration, by analytical differentiation of all the governing discrete boundary layer equations [4.4 - 4.6].

The advantage of analytically determining the Jacobian matrix is that it avoids discretisation errors, which can slow down or even stall convergence. It also saves computational effort since the equations to be solved need only be evaluated once per iteration rather than for every combination of equation and unknown, which is at least nine times in this case. This produces a quadratic convergence rate, provided that a sufficiently accurate initial value is 
known and the Jacobian inverse exists. The integral form of the boundary layer equations is used to minimise the number of additional variables and hence the computation time.

\subsubsection{The algorithm for Newton's method for non-linear systems}

To approximate the solution of a non-linear system of $n$ unknowns, $F(x)=0$, is given an initial approximation of $x$, the following procedure is used:

At each step, the $n \times n$ system $J(x) y=-F(x)$ is solved for $y$, where $J(x)_{i, j}=\left(\partial f_{i}(x) / \partial x_{j}\right)$ for $1 \leq i \leq n$ and $1 \leq j \leq n$. Then $x$ is set to $x=x+y$ until $y$ is sufficiently small, which then gives $x$ as the solution to the non-linear system.

The functional iteration procedure evolves from selecting $x^{(0)}$ and generating $x^{(k)}=x^{(k-1)}-J\left(x^{(k-1)}\right)^{-1} F\left(x^{(k-1)}\right)$ for $k=1,2, \ldots, n$.

\subsubsection{The linear system}

In the present application, the vector function $F$ referred to includes only the boundary layer equations. These equations will be solved in the form of a linear system $J(x) y=-F(x)$ using Gaussian elimination with partial pivoting (Burden and Faires, 2005). Gaussian elimination with partial pivoting helps to reduce round-off errors, since it will demand that some rows should interchange to ensure the pivot element is not zero. In larger systems, it is very difficult to predict in advance when round-off errors can occur.

\subsubsection{The Jacobian}

The Jacobian is defined as:

$$
J\left(\delta^{*}, \theta, U_{e}\right)=\left[\begin{array}{lll}
\frac{\partial f_{1}}{\partial \delta^{*}} & \frac{\partial f_{1}}{\partial \theta} & \frac{\partial f_{1}}{\partial U_{e}} \\
\frac{\partial f_{2}}{\partial \delta^{*}} & \frac{\partial f_{2}}{\partial \theta} & \frac{\partial f_{2}}{\partial U_{e}} \\
\frac{\partial f_{3}}{\partial \delta^{*}} & \frac{\partial f_{3}}{\partial \theta} & \frac{\partial f_{3}}{\partial U_{e}}
\end{array}\right]
$$

where $f_{1}, f_{2}$ and $f_{3}$ are defined in equations [4.4-4.6].

The values in the Jacobian are as follows: 


$$
\begin{aligned}
& \frac{\partial f_{1}}{\partial \delta^{*}}=-\frac{1}{4} \frac{\partial C_{f, m}}{\partial \delta^{*}}+\frac{1}{2} \frac{1}{U_{e, m}}\left(\frac{U_{e, i}-U_{e, i-1}}{\Delta x_{1, i}}\right) \\
& \frac{\partial f_{1}}{\partial \theta}=\frac{1}{\Delta x_{1, i}}-\frac{1}{4} \frac{\partial C_{f, m}}{\partial \theta}+\frac{1}{U_{e, m}}\left(\frac{U_{e, i}-U_{e, i-1}}{\Delta x_{1, i}}\right)
\end{aligned}
$$

$$
\frac{\partial f_{1}}{\partial U_{e}}=-\frac{1}{4} \frac{\partial C_{f, m}}{\partial U_{e}}+\frac{4}{\Delta x_{1, i}}\left(2 \theta_{m}-\delta_{m}^{*}\right)\left(\frac{U_{e, i-1}}{\left(U_{e, i}+U_{e, i-1}\right)^{2}}\right)
$$

$$
\frac{\partial f_{2}}{\partial \delta^{*}}=\frac{\theta_{m}}{2 \Delta x_{1, i}} \frac{\partial H_{m}^{*}}{\partial \delta^{*}}-\frac{\partial C_{D, m}}{\partial \delta^{*}}+\frac{H_{m}^{*}}{4} \frac{\partial C_{f, m}}{\partial \delta^{*}}+\frac{C_{f, m}}{4} \frac{\partial H_{m}^{*}}{\partial \delta^{*}}
$$

$$
+\left(\frac{U_{e, i}-U_{e_{, i-1}}}{U_{e_{, i}}+U_{e, i-1}}\right)\left(\frac{\partial H_{m}^{*}}{\partial \delta^{*}} \frac{1}{\Delta x_{1, i}}\left(\theta_{m}-\delta_{m}^{*}\right)-\frac{H_{m}^{*}}{\Delta x_{1, i}}\right)
$$

$$
\begin{gathered}
\frac{\partial f_{2}}{\partial \theta}=\frac{H_{i}^{*}-H_{i-1}^{*}}{2 \Delta x_{1, i}}+\frac{\theta_{m}}{\Delta x_{1, i}} \frac{\partial H_{m}^{*}}{\partial \theta}-\frac{\partial C_{D, m}}{\partial \theta}+\frac{H_{m}^{*}}{4} \frac{\partial C_{f, m}}{\partial \theta}+\frac{C_{f, m}}{4} \frac{\partial H_{m}^{*}}{\partial \theta} \\
+\left(\frac{U_{e, i}-U_{e_{i-1}}}{U_{e_{, i}}+U_{e, i-1}}\right)\left(\frac{\partial H_{m}^{*}}{\partial \theta} \frac{1}{\Delta x_{1, i}}\left(\theta_{m}-\delta_{m}^{*}\right)+\frac{H_{m}^{*}}{2 \Delta x_{1, i}}\right)
\end{gathered}
$$

$$
\frac{\partial f_{2}}{\partial U_{e}}=-\frac{\partial C_{D, m}}{\partial U_{e}}+\frac{H_{m}^{*}}{4} \frac{\partial C_{f, m}}{\partial U_{e}}+\frac{4 H_{m}^{*}}{\Delta x_{1, i}}\left(\theta_{m}-\delta_{m}^{*}\right)\left(\begin{array}{c}
U_{e_{i-1}} \\
\left(U_{e, i}+U_{e, i-1}\right)^{2}
\end{array}\right)
$$

$$
\frac{\partial f_{3}}{\partial \delta^{*}}=-\frac{U_{e, m}}{2 \Delta x_{1, i}\left(h_{m}-\delta_{m}^{*}\right)^{2}}\left(\delta_{i}^{*}-\delta_{i-1}^{*}-h_{i}+h_{i-1}\right)+\frac{U_{e, m}}{\Delta x_{1, i}\left(h_{m}-\delta_{m}^{*}\right)}
$$

$$
\frac{\partial f_{3}}{\partial \theta}=0
$$

$$
\frac{\partial f_{3}}{\partial U_{e}}=\frac{1}{2 \Delta x_{1, i}\left(h_{m}-\delta_{m}^{*}\right)}\left(\delta_{i}^{*}-\delta_{i-1}^{*}-h_{i}+h_{i-1}\right)-\frac{1}{\Delta x_{1, i}}
$$

The terms within the Jacobian depend on the shape parameter correlations [3.32 - 3.34], for $C_{f}, C_{D}$ and $H^{*}$. These derivatives were obtained analytically to ensure robustness and efficiency of the overall system. The latter differs from other work. 


\subsubsection{Initial condition}

The local Newton method solved point by point needs an appropriate initial value to converge. In addition, an initial value is needed for the Crank-Nicolson method and the logical choice of zero cannot be used since $C_{f}$ and $C_{D}$ are singular there.

The initial value for displacement thickness $\delta^{*}$ and momentum thickness $\theta$ are set according to the similarity solution of Blasius (Drela, 1985). These are:

$$
\begin{aligned}
& \delta^{*}=1.7208 \sqrt{\frac{v x_{1}}{U_{e}}} \\
& \theta=0.664 \sqrt{\frac{v x_{1}}{U_{e}}}
\end{aligned}
$$

The initial values obtained from this function are a sufficiently close approximation to solve most boundary layer solutions (Drela, 1985). The value of $x_{1}$ is set at the smallest value possible to ensure convergence, while $U_{e}$ is set to the velocity at the first point.

The initial value for $U_{e}$ is set at $U_{e, s p e c}$, which is obtained from the inviscid solver. The velocity function [4.3] is not used away from the singularity; instead $U_{e}$ is set equal to $U_{e, s p e c}$ for $H<2.5$ after which the velocity equation [4.3] is used to solve for $U_{e}$.

\subsection{Inviscid flow solver}

The flow solver is based on the Artificial-Compressibility Characteristic-Based split (CBSAC) scheme (Nithiarasu, 2003; Malan, Lewis and Nithiarasu, 2002; Malan and Lewis, 2011), which is based on the original Chorin split and has similarities with other projection schemes widely employed in incompressible flow calculation. Discretisation along characteristics and a pressure-Poisson equation ensure a stable scheme. It can solve both compressible and incompressible flows using a unified approach.

The scheme essentially contains three steps. In the first step, the intermediate velocity field is established. In the second step, the pressure is obtained from a projected continuity equation. Finally, the intermediate velocities are corrected to get the final velocity values. Any additional scalar equation, such as temperature and concentration, can be added as a fourth step. 
The three steps of the CBS-AC algorithm can be written as:

\section{Step 1: Intermediate momentum}

$$
\Delta \tilde{U}_{i}=\tilde{U}_{i}-U_{i}^{n}=\Delta t\left[-\frac{\partial}{\partial x_{j}}\left(u_{j} U_{i}\right)+\frac{1}{\operatorname{Re}} \frac{\partial \tau_{i j}}{\partial x_{j}}+\frac{\Delta t}{2} u_{k} \frac{\partial}{\partial x_{k}}\left(\frac{\partial}{\partial x_{j}}\left(u_{j} U_{i}\right)\right)\right]^{n}
$$

Where $U_{i}^{n}=U_{i}\left(t_{n}\right), \Delta t=t^{n+1}-t^{n}$ and indicates an intermediate quantity. Further, $t$ and $n$ respectively denote pseudo-solution time and iteration number. The higher-order term is due to time discretisation using the characteristic concept and serves to stabilise the convective term. Viscous terms are included although they are negligible for inviscid flow and the flow solver is not required to resolve the boundary layer.

Step 2: Density or pressure

$$
\begin{aligned}
\Delta \rho & =\rho^{n+1}-\rho^{n}=\left(\frac{1}{c^{2}}\right)^{n} \Delta p=\left(\frac{1}{c^{2}}\right)^{n}\left(p^{n+1}-p^{n}\right) \\
& =-\Delta t\left[\frac{\partial U_{i}^{n}}{\partial x_{i}}+\theta_{1} \frac{\partial \Delta \tilde{U}_{i}}{\partial x_{i}}-\Delta t \theta_{1}\left(\frac{\partial^{2} p^{n}}{\partial x_{i} \partial x_{i}}+\theta_{2} \frac{\partial^{2} \Delta p}{\partial x_{i} \partial x_{i}}\right)\right]
\end{aligned}
$$

Step 3: Momentum correction

$$
\Delta U_{i}=U_{i}^{n+1}-U_{i}^{n}=\Delta \tilde{U}_{i}-\Delta t \frac{\partial p^{n+\theta_{2}}}{\partial x_{i}}
$$

Where $0.5 \leq \theta_{1} \leq 1$ and $0 \leq \theta_{2} \leq 1$. For the explicit scheme, $\theta_{2}=0$ is employed (Nithiarasu, 2003). Due to the artificial compressibility formulation, a finite value of $c^{2}$ may be used.

\subsubsection{Mesh generation}

Before a numerical solution for a physical process can be obtained, a grid must be constructed. The physical domain is covered with a mesh to identify discrete volumes or elements where conservation laws can be applied. A well-constructed mesh greatly improves the quality of the solution (Tannehill et al., 1997). Generation of grids can be either structured or unstructured.

In the case of the boundary layer, cell spacing normal to the wall must be very fine (proportional to $\sqrt{\mathrm{Re}}$ ) in order to capture the sharp gradients in this direction. The use of a structured scheme allows a cell shape that is elongated in the flow direction. Alternatively, 
the use of unstructured grids, for example, triangles in a two-dimensional problem, requires a higher cell density in the boundary layer since the cells need to be as nearly equilateral as possible to avoid grid-induced errors in the solution (Tannehill et al., 1997).

These are typical mesh generation problems when solving the viscous effects with the Navier-Stokes equations. However, these problems can be avoided by using boundary layer modelling, since it does not require mesh generation at all in the boundary layer.

\subsection{Mesh moving algorithm}

The mesh movement routine is a simple interpolation function

$$
\underline{\delta}=r \underline{\delta_{1}}+(1-r) \underline{\delta_{2}}
$$

where

$$
r=\frac{d_{2}^{3 / 2}}{\left(d_{1}^{3 / 2}+d_{2}^{3 / 2}\right)}
$$

In this case, $\delta$ is the displacement of the grid point from its original position, $d_{1}$ and $d_{2}$ are the closest distances from that point to the two closest boundaries and $\underline{\delta_{1}}$ and $\underline{\delta_{2}}$ are the displacements of those two boundary points. This might seem like a crude approach but it is sufficient for small displacements in aerodynamic applications and is selected for its negligible computational cost.

\subsection{Coupling and interaction method}

The interaction method between the solver and the different routines to calculate the boundary and move the mesh happens as follows:

First, the CFD solver lets the flow solution converge to a point where the residual is less than the specified tolerance divided by some factor, for example, 2. This factor is simply intended to prevent an excessive number of mesh movements as convergence is approached, since every time the mesh is moved, the residual increases slightly. Therefore, it is desirable to decrease the residual more than necessary to ensure that even after the mesh is moved, the system is still converged to the required tolerance.

Second, the solver uses the boundary layer thickness obtained from the boundary layer solution to move boundary node $i$ as follows:

$$
\underline{x}_{i}^{(n)}=\alpha \delta_{i}^{*} \underline{n}+(1-\alpha) \underline{x}_{i}^{(n-1)}
$$


Where $\underline{x}_{i}^{(n)}$ and $\delta \underline{x}_{i}^{(n-1)}$ are the new and previous displacements of node $i$ from its original position, $\delta^{*}$ is the computed boundary layer thickness, $\underline{n}$ is a unit vector normal to the boundary and $\alpha$ is an under-relaxation coefficient.

Coupling of the inviscid and viscous flow requires an under-relaxation parameter $\alpha$ to ensure stability of the numerical system. Essentially, the under-relaxation parameter only moves the wall coordinates a fraction of the whole movement that is needed to move to the new position specified by the boundary layer solver. It is a number between zero and one. If it is too close to one, stability is sacrificed, whereas when it is close to zero, the system is very stable but takes longer to converge. Ideally, the under-relaxation parameter is set as close to one as possible without having numerical instabilities.

Following the mesh movement step, the mesh is repreprocessed and the residual is calculated again. If this residual is less than the convergence tolerance, the program will give the resulting output, otherwise it restarts from the beginning, repeating the process.

Preprocessing of a mesh for a finite volume method means calculating the volumes and edge coefficients of dual cells. When the mesh is moved, this information changes and must be recalculated. The boundary condition required for the inviscid solver at a solid wall is the specification of the surface streamline position. This is equal to the displacement thickness $\delta^{*}$ calculated by the boundary layer routine.

\subsection{Laminar wake singularity}

The velocity equation [4.3] (the discrete version of equation [3.47]) involves the fictitious 'channel height' $h$ calculated as per equation [3.40]. As stated at the end of Section 3.5.3, the selection of a smaller value of $\dot{V}$ in equation [3.40] is more effective at removing the singularity, but leads to computed velocities which differ markedly from the specified values,

and hence an inaccurate solution of the displacement thickness. Larger values of $\dot{V}$ give more accurate solutions, but less robustness against the Goldstein singularity.

In practice, it was found that an unacceptable amount of accuracy had to be sacrificed in order for the iterative process to be robust in all situations. For example, the early cycles of computing the boundary layer around the airfoil, where the initially imposed velocity distribution is the inviscid solution with a large adverse pressure gradient near the trailing edge, are particularly problematic. 
To solve this unacceptable loss of accuracy, the function $\delta_{\text {estimate }}^{*}$ is set to the previously computed $\delta^{*}$ at each iteration (being set to zero, initially). $\dot{V}$ can then be set to a small value to ensure robust solution, while at convergence, the velocities computed from equation [4.3] approach the imposed values and so no accuracy is sacrificed. The $\dot{V}$ selected is $0.005 \mathrm{~m}^{3} . \mathrm{s}^{-1}$. It was found that for this iterative process to converge, it had to be 'frozen' at some stage. To achieve this, the maximum percentage change in $\delta^{*}$ over the entire boundary layer is monitored and when it falls below a set threshold, here set to $0.2 \%, \delta_{\text {estimate }}^{*}$ is no longer updated.

To accommodate the wake behind an airfoil, a function was scaled to an estimate of the expected wake function. This was necessary since calculation of the boundary into the wake was not considered in this study. The function fitted was:

$$
\delta_{\text {wake }}^{*}\left(x_{1}\right)=10^{A-3.2\left(x_{1}-1\right)}
$$

where $A$ is selected so that $\delta_{\text {wake }}^{*}(1)=\delta^{*}(1)$. The trailing edge is at $x_{1}=1$.

The function uses the last point before the end of the airfoil and scales the rest of the displacement thickness values moving into the wake accordingly. Since this does not accurately portray the shape of the wake, the approximation function [4.24] will cause a sacrifice of accuracy in the displacement thickness results through and beyond the trailing edge into the wake. Accurate representation of the wake requires implementation of different correlations (Katz and Plotkin, 2001), but otherwise is essentially the same as the process followed for the boundary layer.

\subsection{Implementation into the code}

The boundary layer code is $\mathrm{C}++$ based, since this coding language is computationally expedient and widely used. $\mathrm{C}++$ has a few advantageous characteristics compared with other programming languages. Three of these are applicable; its capabilities of speed, modular programming and its compatibility with $\mathrm{C}$ codes. Modular programming refers to the capability of compiling different source code files separately and then linking them together. This also allows the code to be linked to other languages. 
This approach to solve the boundary layer as a modular system, implies that it can be integrated into any flow solver. It does not have to solve the inviscid flow together with the boundary layer as one monolithic system. Also, the inviscid flow solver needs no knowledge or extra parameters to understand or compute a fictitious transpiration velocity boundary condition.

The only input the boundary layer code requires from the inviscid solver is the coordinates of the nodes as well as the velocities at those nodes. It then calculates the displacement thickness, auxiliary velocity and momentum thickness. The displacement thickness is then returned to the inviscid code and used to calculate the new node point to which the mesh has to move before the whole process starts again.

\subsection{Chapter summary}

This chapter discussed the procedure followed to solve the boundary layer flow and the interaction with the outer inviscid flow. The discretisation of the governing equations was described as well as the interactive procedure by which it is coupled to the inviscid flow solver. A local Newton method is used to solve the boundary layer flow, point by point, by obtaining information about the velocity outside the boundary. The mesh is then moved to the calculated boundary thickness and the process is repeated until convergence is reached. The boundary condition at the edge of the boundary thickness is set to a slip condition. An underrelaxation parameter ensures stability in the coupling condition. An additional velocity equation adjusts the mesh movement to avoid the Goldstein singularity, which is encountered before the trailing edge.

Chapter 5 follows with the results and discussion of the computational performance. 


\section{CHAPTER 5}

\section{VERIFICATION AND VALIDATION}

\subsection{Introduction}

Numerical error in the calculations in numerical fluid dynamics codes should be estimated and then considered to make sure the solution is an accurate representation of reality. First, the concepts of verification and validation are defined. Verification is 'solving the equations right', which means checking for errors introduced by discretisation and coding errors. Validation is 'solving the right equations', meaning checking for modelling errors (Roache, 1997). First, the code is compared with analytical similarity solutions of the Blasius and Falkner-Skan equations without inviscid interaction with the solver. After this has been proven to be accurate, the code is combined with the inviscid solver for further verification. For this purpose, incompressible viscous flow is modelled over a NACA0012 airfoil, and the results compared with those generated by the 'XFOIL' code (Drela and Youngren, 2001).

\subsection{Results without inviscid (outer) flow interaction}

\subsubsection{Steady incompressible laminar flow over a flat plate}

The first test case involved obtaining the Blasius solution of flow over a flat plate. For this purpose, the external velocity was set constant at $1 \mathrm{~m} . \mathrm{s}^{-1}$. The kinematic viscosity used in these test cases was $1 \times 10^{-5} \mathrm{~m}^{2} \cdot \mathrm{s}^{-1}$ with Reynolds numbers going up to $4 \times 10^{5}$. A grid independence study was performed to assess the order of accuracy. For this purpose, four meshes were employed, with mesh spacing $\left(\Delta x_{1}\right)$ varying from 0.267 to 0.01 . 


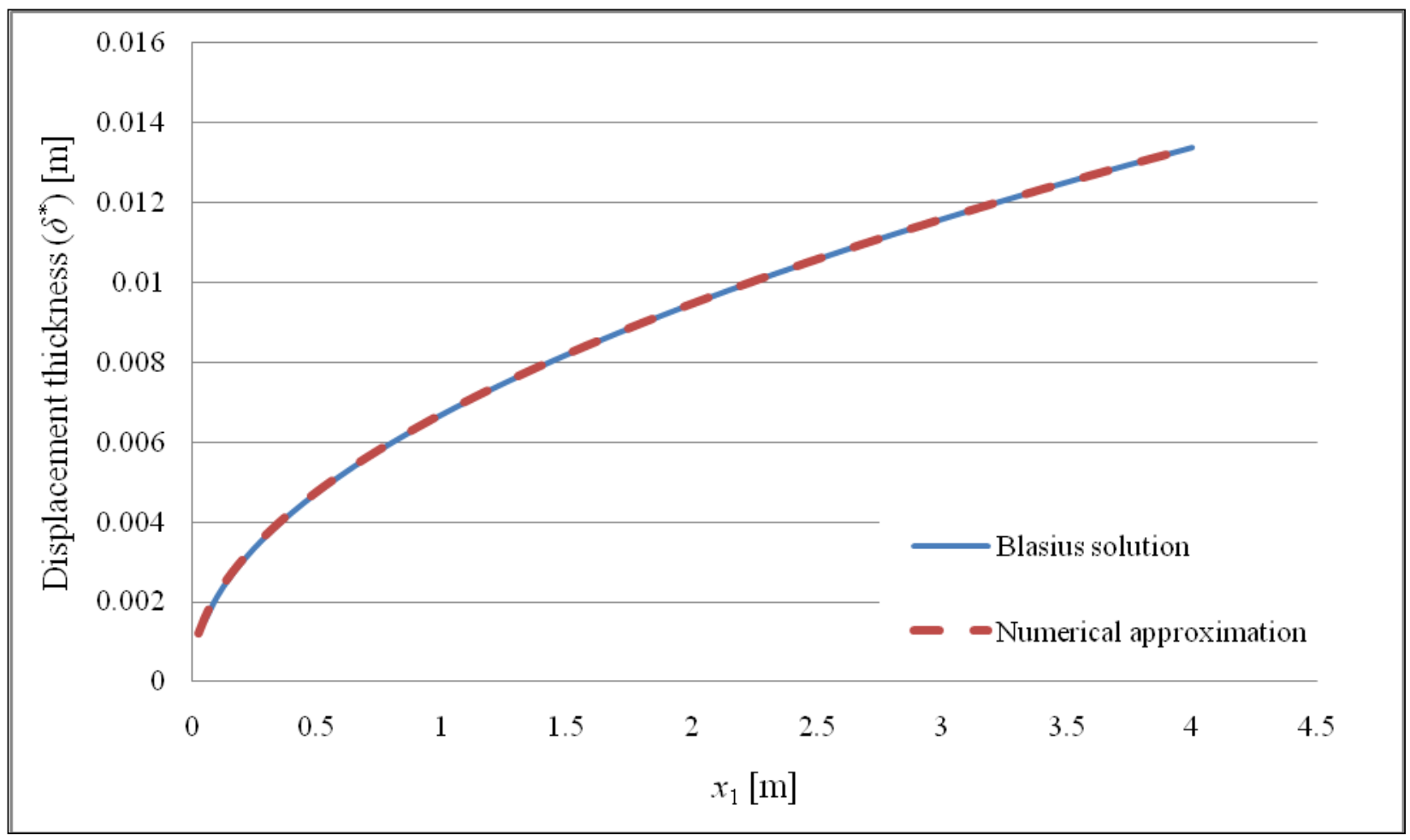

Figure 7: Comparison between the Blasius solution and the numerical solution $\left(\Delta x_{1}=0.05\right)$

The average error between the Blasius similarity solution and the two-integral solution was calculated using equation [5.1].

$$
\text { Error }=\left(\sum_{i=1}^{n}\left|\delta_{i, \text { similarity }}^{*}-\delta_{i, \text { computed }}^{*}\right| / \delta_{i, \text { similarity }}^{*}\right) / n
$$

where $n$ is the number of grid points. The solution achieved on the second-coarsest mesh is compared with the analytical solution in Figure 7, with the errors for all cases given in Table 1 .

\begin{tabular}{|c|c|c|}
\hline & Grid spacing $\left(\Delta x_{1}\right)$ & Average error (\%) \\
\hline Case 1 & 0.267 & $42.46 \%$ \\
\hline Case 2 & 0.05 & $2.06 \%$ \\
\hline Case 3 & 0.0267 & $0.55 \%$ \\
\hline Case 4 & 0.01 & $0.083 \%$ \\
\hline
\end{tabular}

Table 1: Comparison between different mesh sizes for a flat plate

Figures 8 shows the convergence rate of the approximation to the exact Blasius solution when solved with different grid spacings. The Crank-Nicolson scheme has a quadratic convergence rate, which is evident by the slope value of about 2 . 


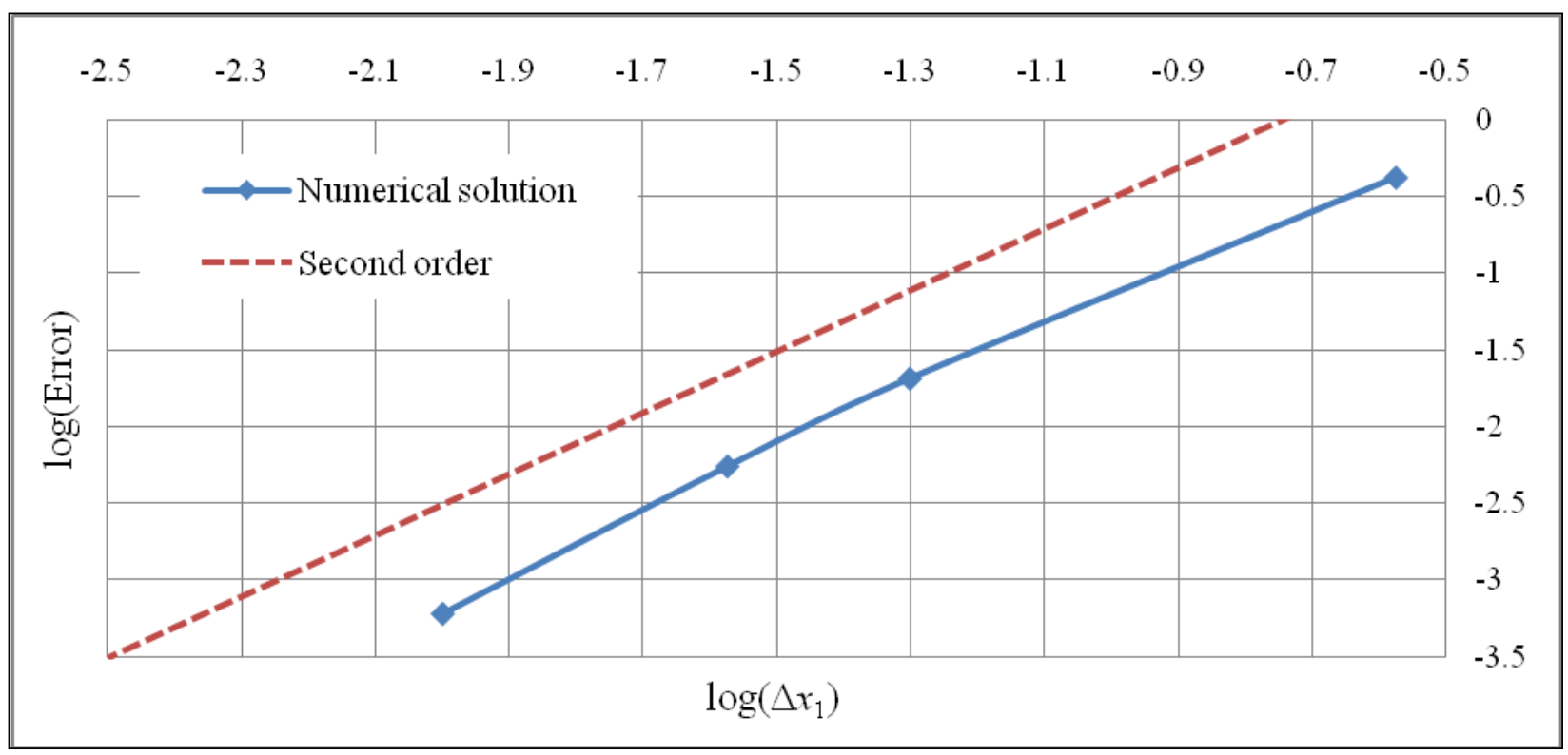

Figure 8: Convergence rate for the Blasius solution using different grid spacings with the dashed line depicting formal second-order accuracy.

\subsubsection{Incompressible laminar flow over an inclined plate}

The aforementioned testcase was next extended to a plate at incidence to the flow, with the free-stream velocity remaining $1 \mathrm{~m} . \mathrm{s}^{-1}$. Exact solutions were obtained by solving the FalknerSkan equation. The displacement thickness evolution for various angles of attack is depicted in Figure 9. As in the case with the flat plate, different mesh sizes were tested and a finer mesh spacing was found to give more accurate results. Table 2 shows the grid convergence of two of the angled plate results, where $\pi \beta / 2$ is the angle of the plates from the horizontal (see Figure 2 in Section 2.1). The results demonstrate that the quadratic convergence of the CrankNicolson method has again been realised.

\begin{tabular}{|c|c|c|}
\hline & Step size $\left(\Delta x_{1}\right)$ & Error (\%) \\
\hline $\boldsymbol{\beta}=\mathbf{0 . 3}$ & 0.05 & $2.47 \%$ \\
\hline & 0.02 & $0.35 \%$ \\
\hline $\boldsymbol{\beta}=\mathbf{0 . 4}$ & 0.01 & $0.082 \%$ \\
\hline & 0.05 & $2.11 \%$ \\
\hline & 0.02 & $0.23 \%$ \\
\hline & 0.01 & $0.065 \%$ \\
\hline
\end{tabular}

Table 2: Comparison between different mesh sizes for angled plates 
Figure 9 shows the calculated displacement thickness for various plate angles. The numerical and similarity solutions all agree to plotting accuracy.

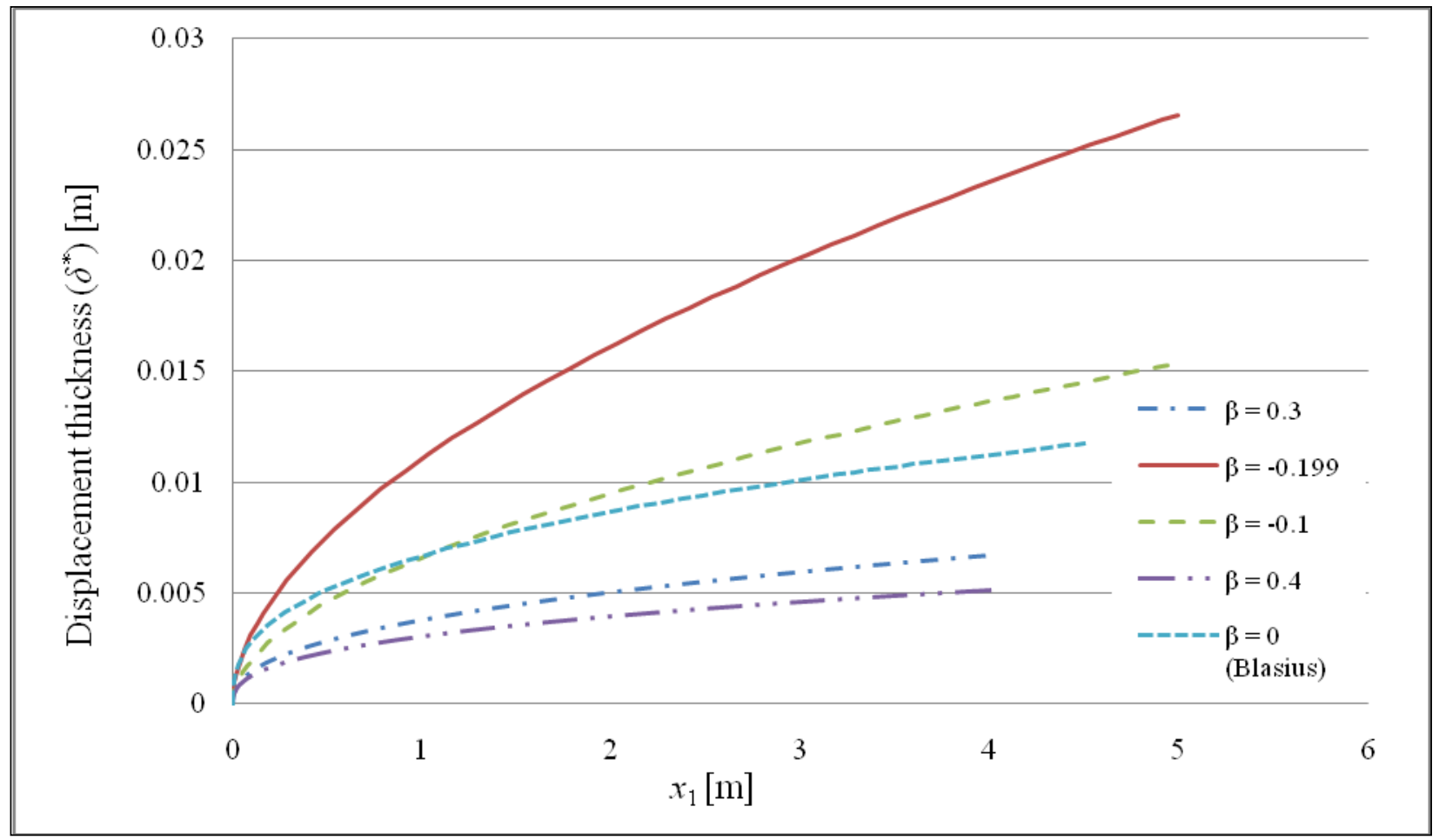

Figure 9: Displacement thicknesses of flow over an inclined plate for various angles of attack

\subsection{Results when interacting with inviscid outer flow}

\subsubsection{Incompressible laminar flow over a flat plate}

Having successfully validated the boundary layer modelling technology, viscous-inviscid interaction problems were considered next. The first problem was again the laminar flow over a flat plate at zero incidence. A structured mesh was employed as depicted in Figure 10. The boundary condition at the leading edge of the flat plate was set to a fixed inflow velocity parallel to the plate. The boundary condition at the trailing edge was set to an outflow condition whereby velocity is extrapolated from inside the flow domain while pressure was unconstrained. The boundary condition at the top of the domain was set to the fixed freestream velocity. 


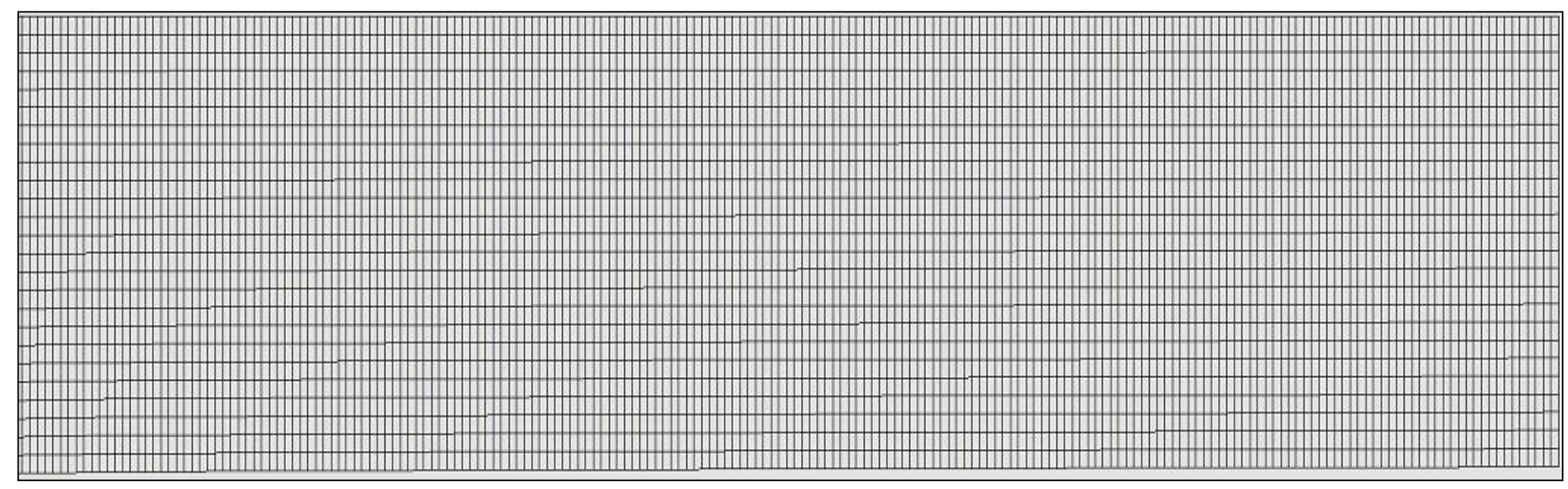

Figure 10: Flat plate showing structured mesh

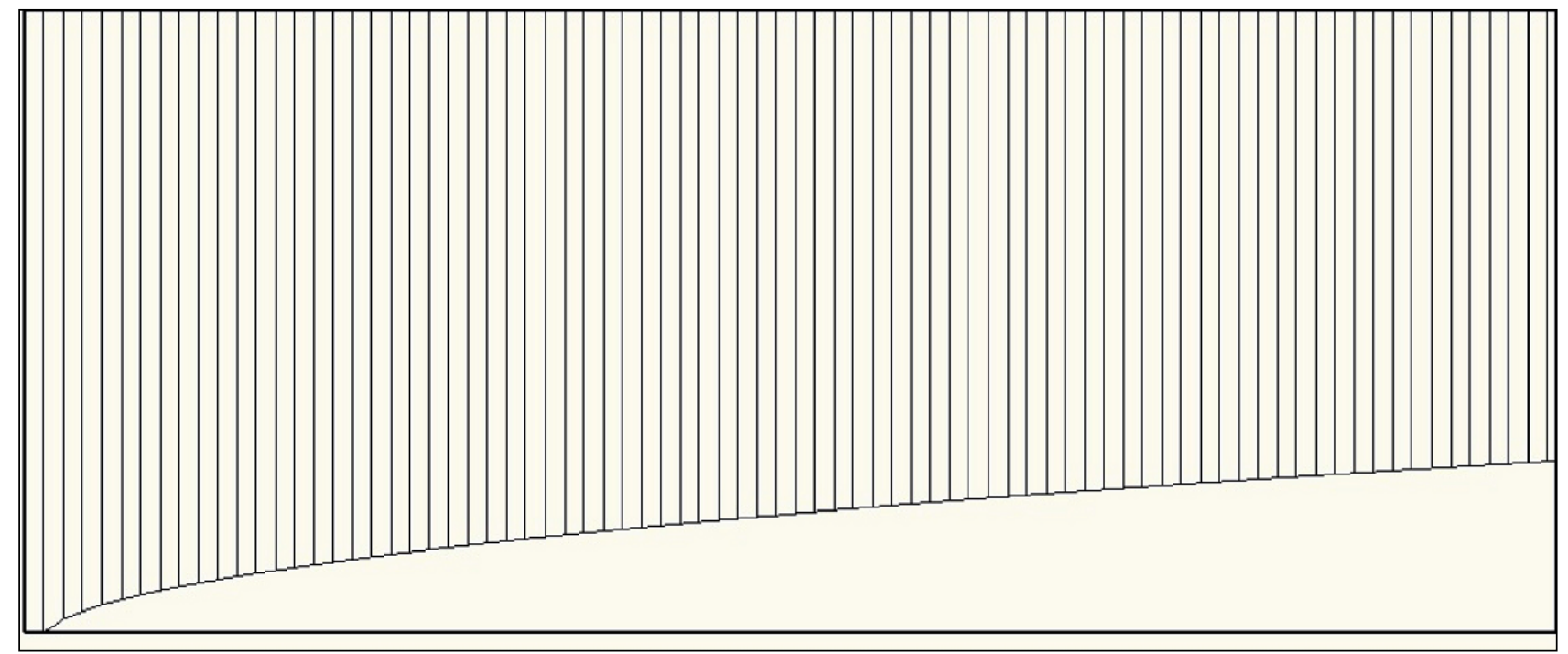

Figure 11: Close-up of mesh movement for flat plate flow in Figure 11, magnified 200 times in $x_{2}$-direction

The predicted magnified boundary layer displacement mesh is depicted in Figure 11. The structured mesh consists of 5226 nodes with $\Delta x_{1}=0.02 \mathrm{~m}$ and the solver converged to a solution with an error percentage of $0.2 \%$ (Equation [5.1]). The percentage error is less than the uncoupled case where the error was approximately $0.55 \%$ for a similar step size. The solver was found to be stable and robust, with only two tuneable parameters to be set: the under-relaxation parameter $\alpha$ (see equation [4.23]), which affects the iterations between the inviscid and boundary layer solver, and the CFL number of the inviscid solver. In this case, $\alpha$ was set to 0.7 , and the CFL number was set to 0.9 . The solver was run on eight Intel Xeon CPUs of $2.33 \mathrm{GHz}$ each and required 30.3 seconds to converge.

\subsubsection{Incompressible laminar flow over an inclined plate}

Three different inclined plates were used to further test the interaction of the solver with the boundary layer code. The angles were selected by choosing a few $\beta$ values, which correlated with an angle of attack range that resulted in positive pressure gradients, i.e. no flow 
separation. $\beta$ values of $0.2,0.3$ and 0.4 were selected which give $18^{\circ}, 27^{\circ}$ and $36^{\circ}$ respectively. Unstructured meshes were employed consisting of $2355\left(\Delta x_{1}=0.0625 \mathrm{~m}\right)$, $2190\left(\Delta x_{1}=0.02 \mathrm{~m}\right)$ and $1426\left(\Delta x_{1}=0.02 \mathrm{~m}\right)$ nodes, respectively. The unstructured mesh for the $36^{\circ}$ test case is shown in Figure 13.

To obtain a meaningful solution, velocity was to be prescribed at the outer boundary. As there is no clear difference between in- and outflow regions, the analytical velocity was imposed across the entire outer boundary (with pressure being solved for). The final numerical solution for the displacement thickness from the two-integral method was tested against the similarity solution of Falkner-Skan to verify the results obtained. This is shown in Figure 12 , indicating that an accurate solution was obtained.

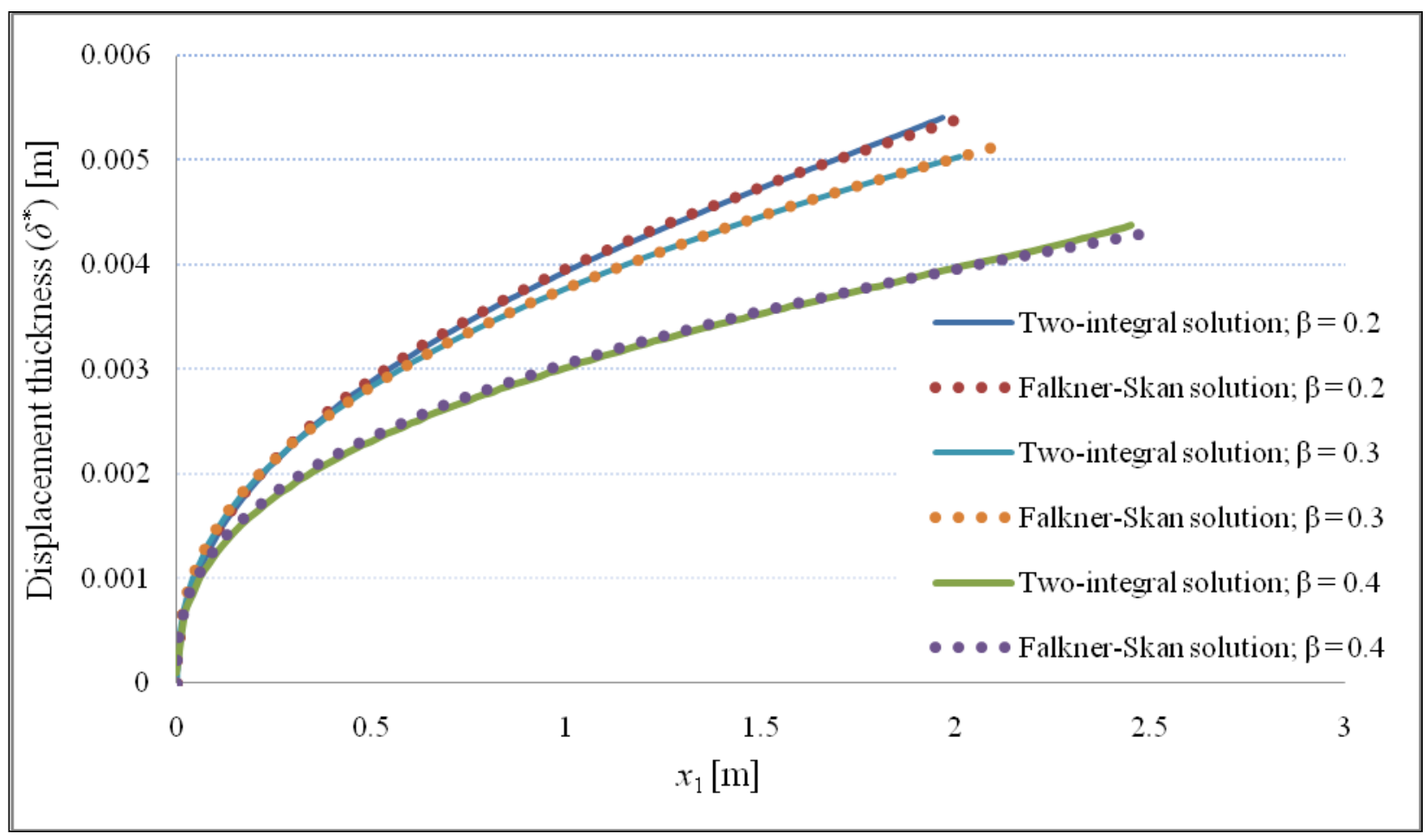

Figure 12: Comparison between the two-integral solution and the Falkner-Skan similarity solution

Table 3 shows the statistics for the iterations needed to converge to a coupled solution as well as the accuracy and the under-relaxation parameter used. The error was calculated as before using equation [5.1]. The accuracies achieved are similar to those of the pure boundary layer modelling case previously, demonstrating the accuracy of the developed viscous-inviscid interaction methodology. 


\begin{tabular}{|c|c|c|c|}
\hline $\boldsymbol{\beta}$ & $\begin{array}{c}\text { Coupled } \\
\text { iterations } \\
\text { required }\end{array}$ & $\begin{array}{c}\text { Under-relaxation } \\
\text { parameter }\end{array}$ & $\begin{array}{c}\text { Error [\%] (Viscous-inviscid } \\
\text { interaction method compared with } \\
\text { Falkner-Skan solution) }\end{array}$ \\
\hline 0.2 & 16 & 0.5 & $0.7 \%$ \\
\hline 0.3 & 16 & 0.5 & $0.9 \%$ \\
\hline 0.4 & 18 & 0.5 & $1.2 \%$ \\
\hline
\end{tabular}

Table 3: Numerical statistics surrounding convergence as shown in Figure 12.

Pressure and velocity results are shown for the cases of an angle of $36^{\circ}$ in Figure 13 . The results for the other angles are similar. The solver converged to a stable solution within 14.9 seconds. The under-relaxation coefficient $\alpha$ was set to 0.5 for the angled plate cases, with the CFL number still set to 0.9 . The velocity distribution is shown in Figure 14 using velocity vectors. Figure 15 shows the magnified movement of the mesh along the slope of the domain.

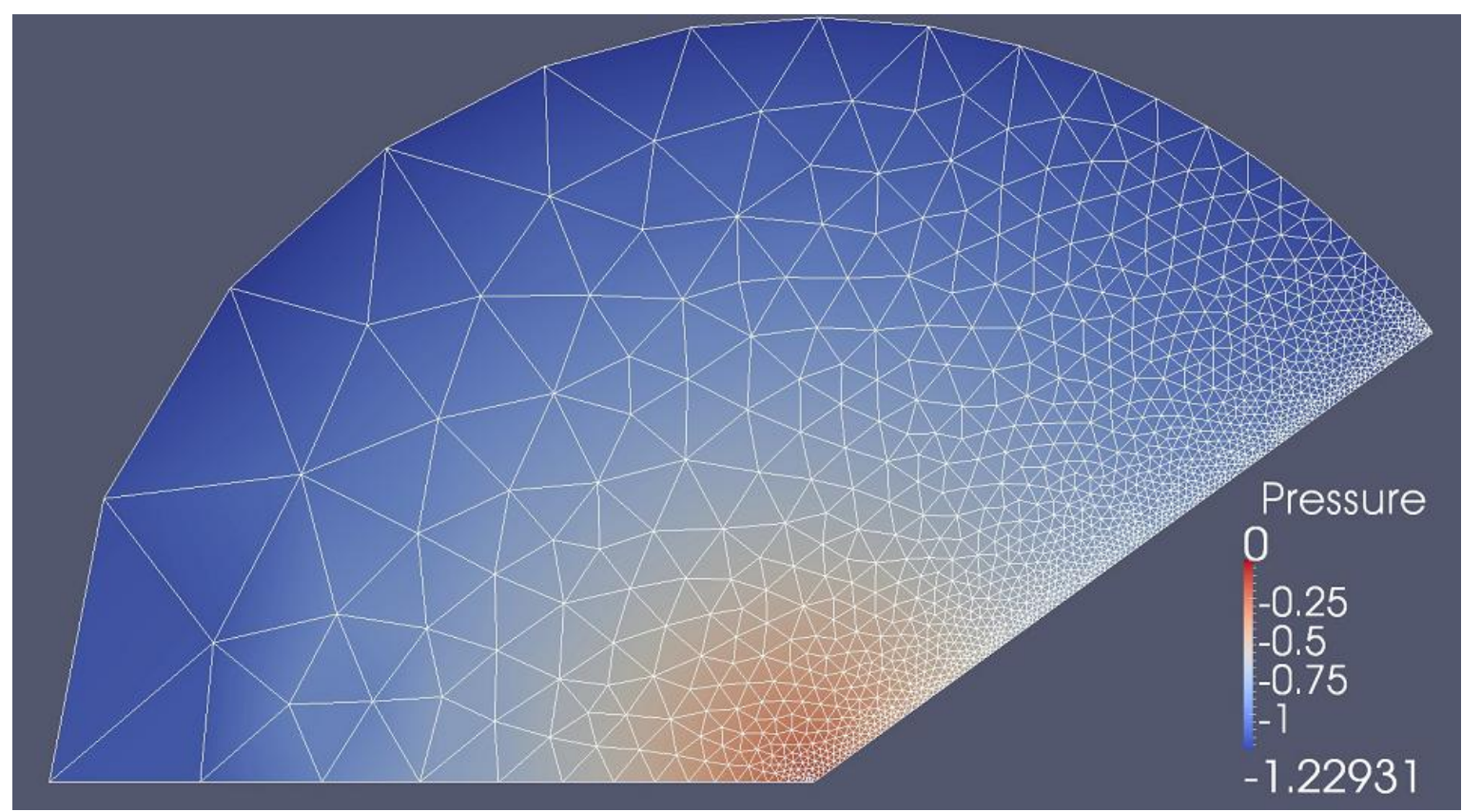

Figure 13: Pressure contours and unstructured mesh $(\beta=0.4)$. Pressure values are in Pa 


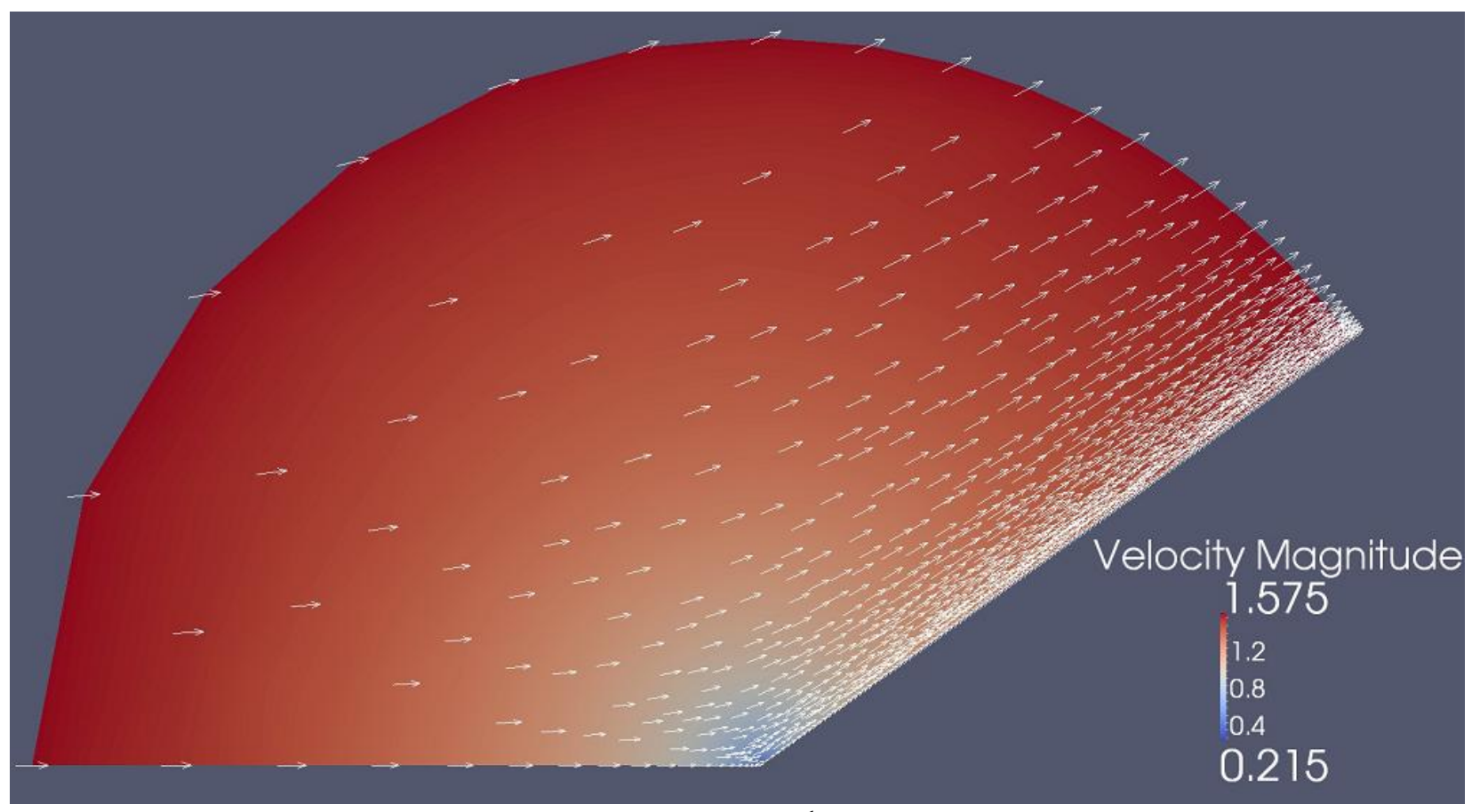

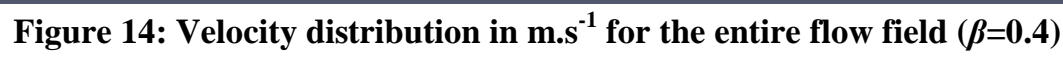

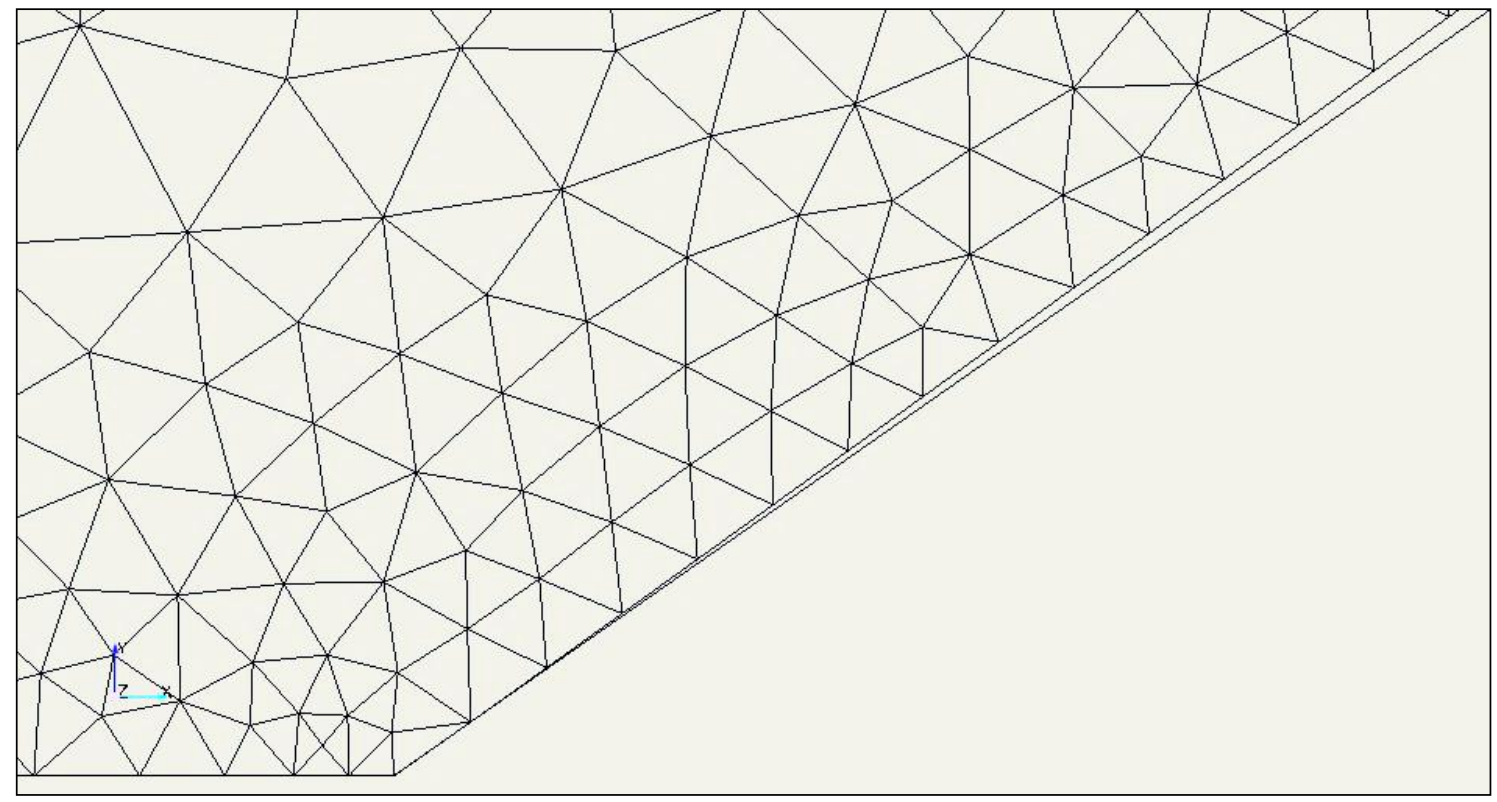

Figure 15: Mesh movement on the $\beta=0.4$ slope mesh, magnified 30 times

\subsubsection{Incompressible laminar flow over a NACA0012 airfoil}

As a concluding example, the flow over a symmetrical airfoil (NACA0012) at zero angle of attack and $\mathrm{Re}=10000$ (laminar) was calculated. Results obtained are compared with simulations of the same airfoil from XFOIL 6.9, a code developed by Drela and Youngren (2001), to assess accuracy of the boundary layer code. In XFOIL, a two-equation integral boundary layer formulation describes the boundary layer and wake. It is strongly interacted with the incompressible potential flow via the surface transpiration model. The system is 
then solved using a full-Newton method, solving the whole flow field simultaneously (Drela and Youngren, 2001), which differs from the method developed in this work. Further noting that XFOIL is today a widely used commercial package, its results serve as meaningful validation.

An unstructured computational mesh consisting of 12064 nodes was employed (as shown in Figure 20). The boundary conditions were set to slip velocity all around the domain. The solver converged to a stable solution with an under-relaxation parameter of 0.1 and converging to a solution within 72 viscous-inviscid iterations in 165 seconds using eight Intel Xeon CPUs of $2.33 \mathrm{GHz}$ each. The inviscid solver CFL number was maintained at 0.9.

Figure 16 shows the solution of the displacement thickness at different stages of convergence. A slight inaccuracy in the vicinity of the trailing edge is evident, where the maximum disparity between the codes of $13.2 \%$ occurs. The line name 'intermediate' refers to a point in convergence where there is a $10 \%$ difference between $U_{e}$ and $U_{e, s p e c}$ (velocity obtained from the inviscid solution). The velocity equation [4.3] suggested overcomes the Goldstein singularity existing at the point of shear stress vanishing but a proper solution of the wake would be required to ensure accurate calculation of the displacement thickness into the wake, whereas in this work, a wake function was used to simulate the displacement thickness across the trailing edge into the wake, as described in Section 4.7. The average difference in predicted displacement thickness between the developed technology and that of XFOIL using equation [5.1], is $3.95 \%$. Figure 17 shows the velocity solution for the flow over the NACA0012 airfoil. Both boundary layer thickness and boundary layer momentum thickness $\left(\delta^{*}\right.$ and $\theta$ ) grow towards the trailing edge and then slowly decrease in the near wake. Figure 18 depicts the shape factor $(H)$ during different stages of convergence. The shape factor begins at a value slightly lower than the Blasius value $(H=2.59)$ and grows gradually towards the trailing edge. Recall that the singularity occurs at $H=4$. 


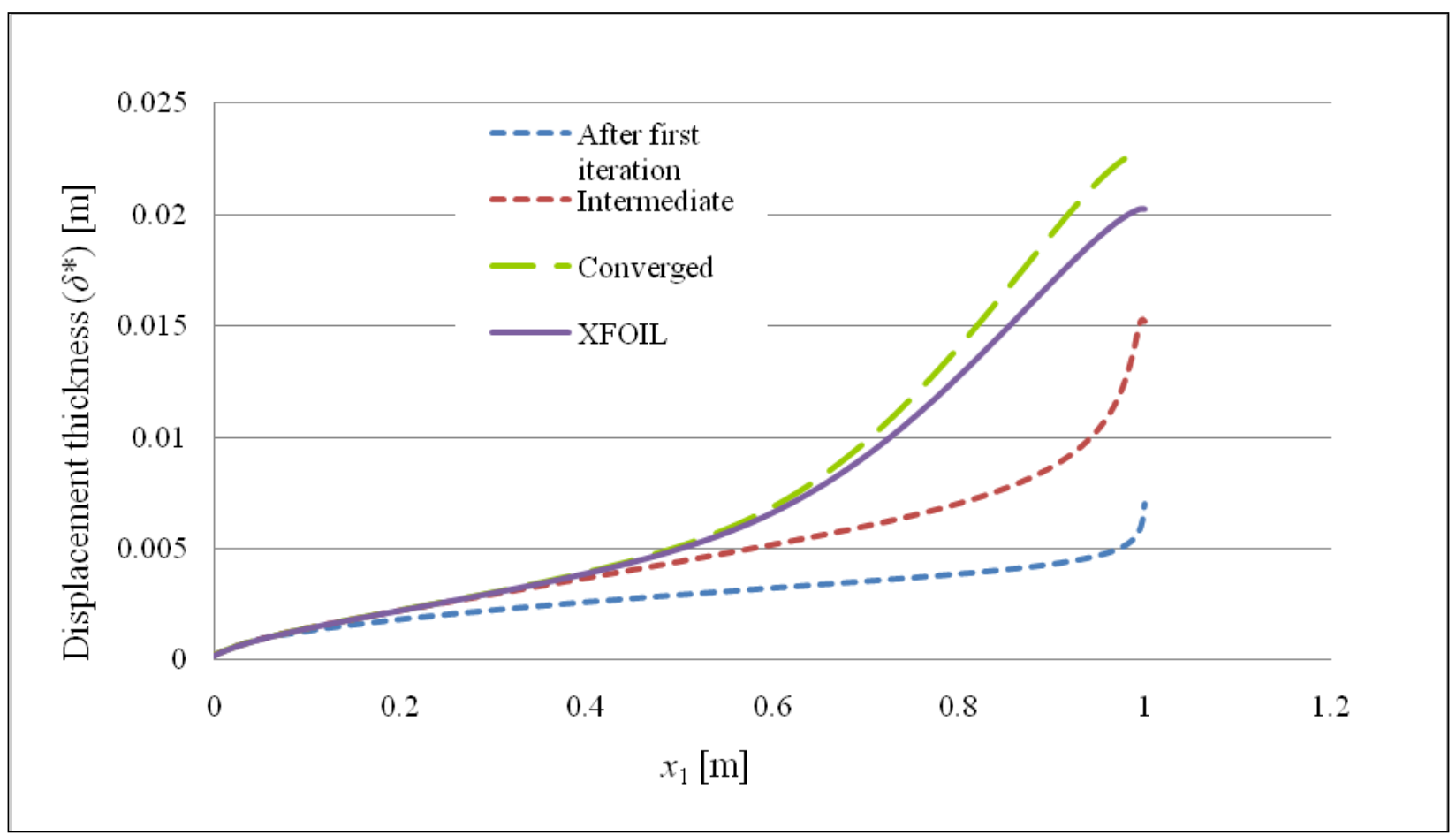

Figure 16: Comparison of the displacement thickness at different stages of convergence for the NACA0012 airfoil

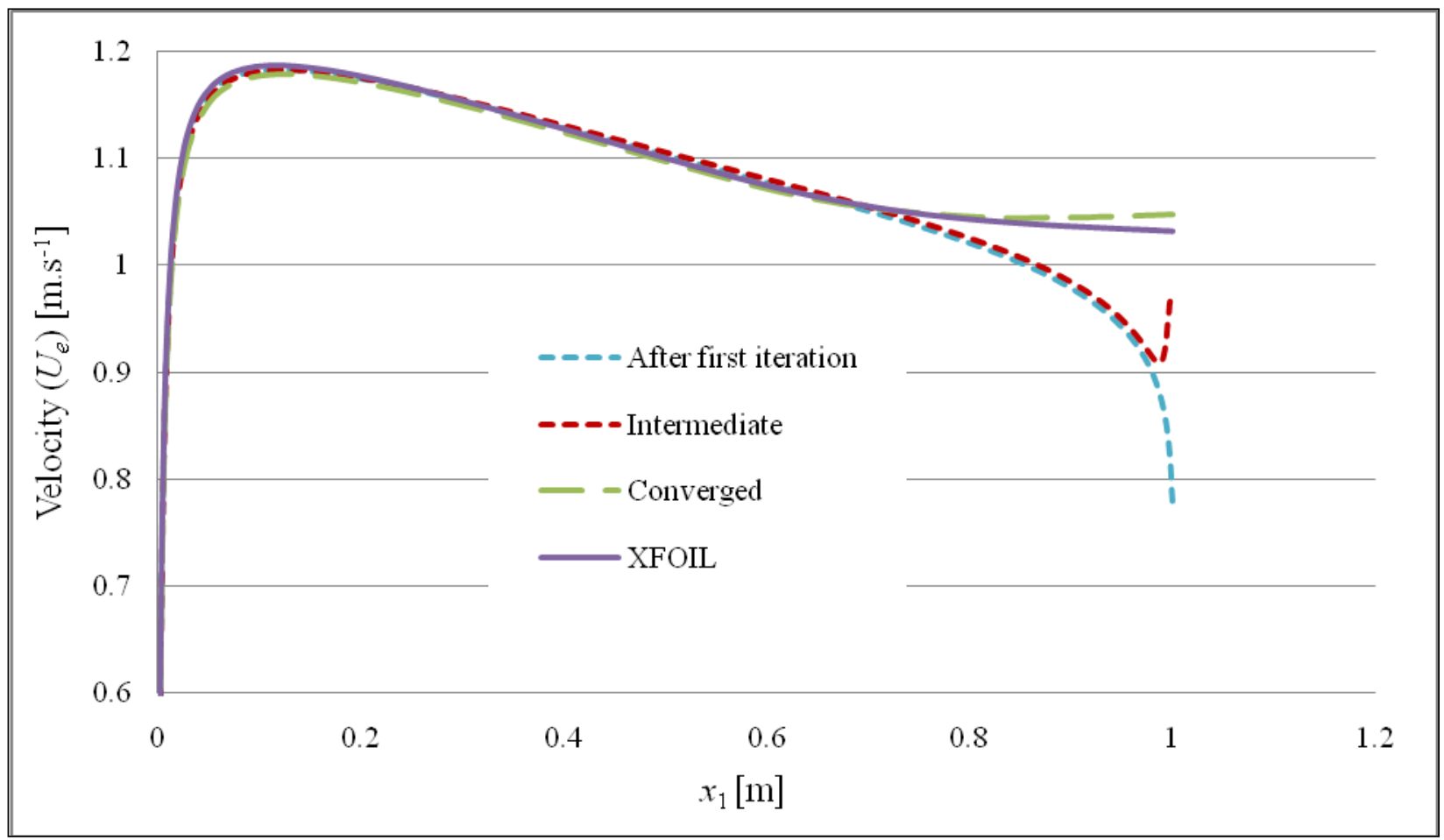

Figure 17: Comparison of velocity distribution for the NACA0012 airfoil 


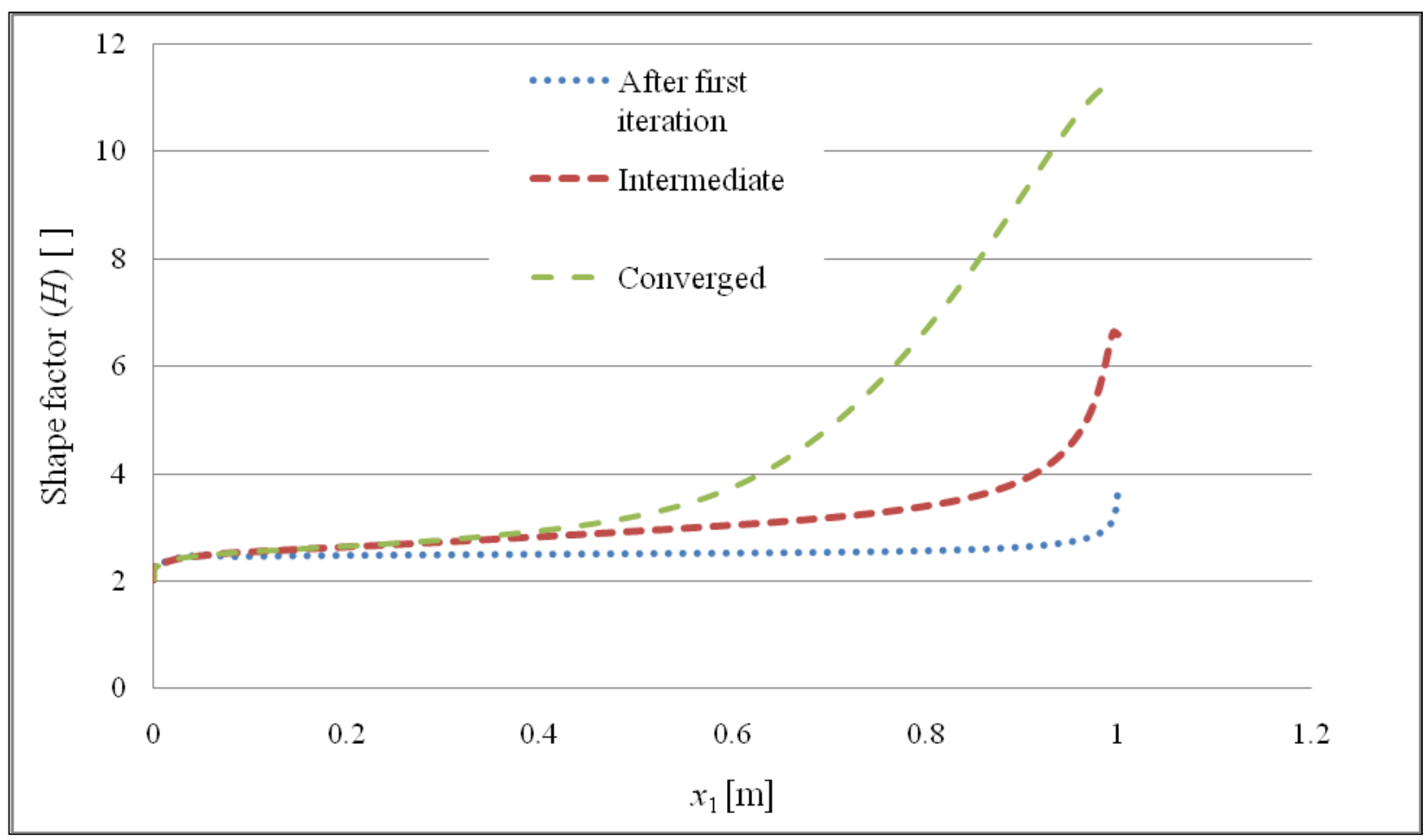

Figure 18: Shape factor at different stages of convergence

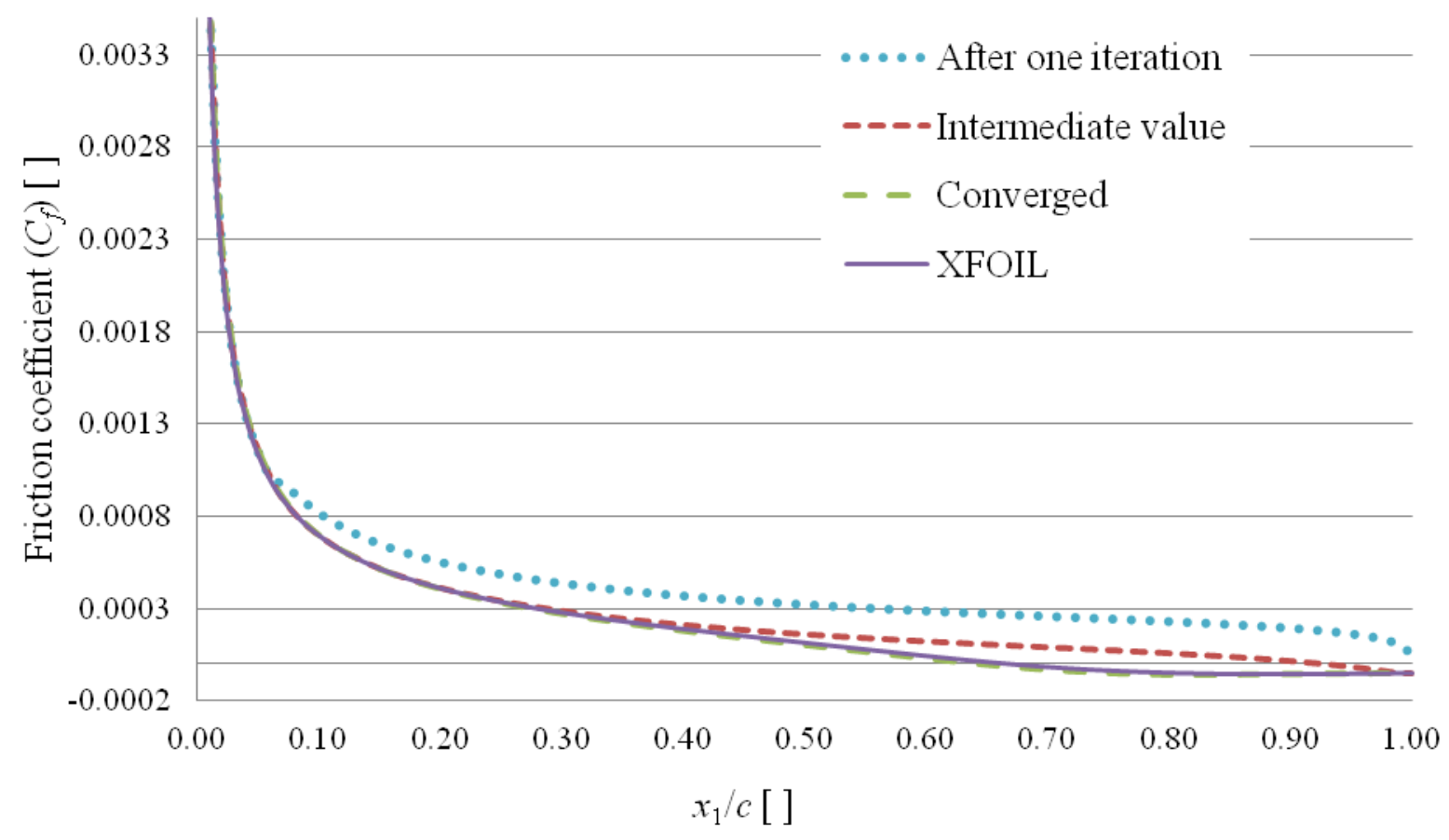

Figure 19: Skin friction coefficient at different stages of convergence 

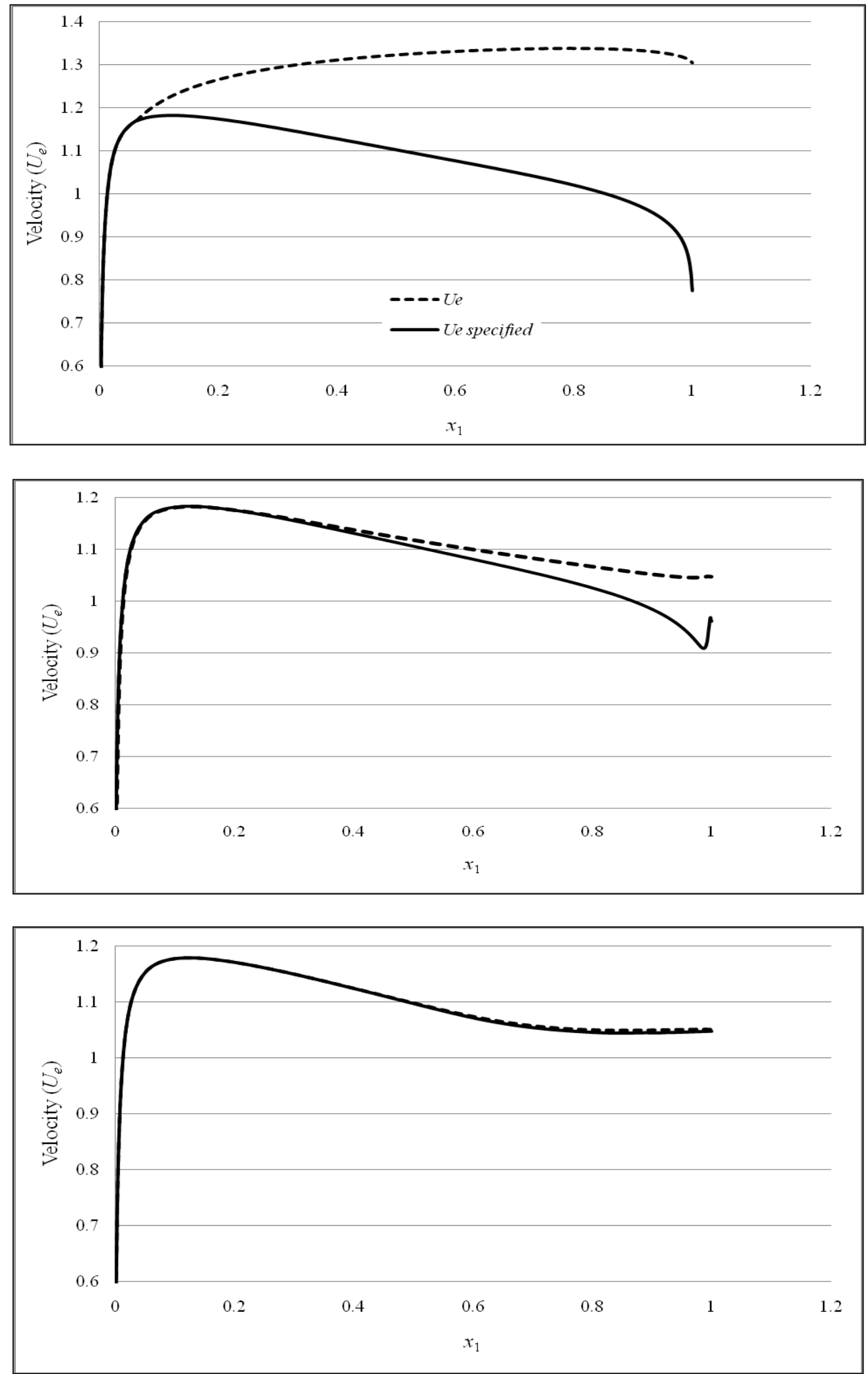

Figure 20: Comparison between the velocity specified and the velocity obtained from the boundary layer solution, after first iteration (top), intermediate (middle) and converged (bottom) 

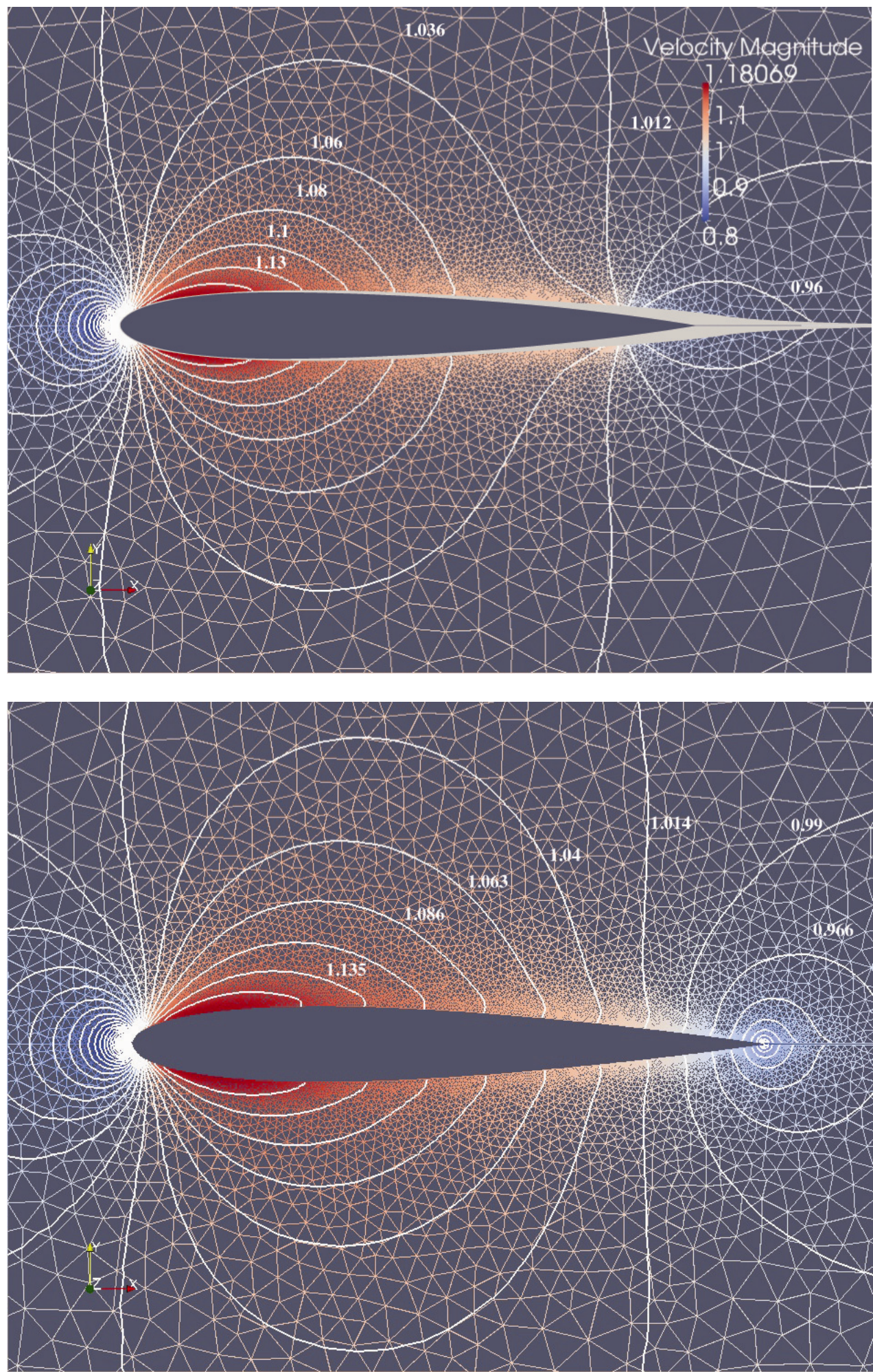

Figure 21: Mesh and velocity contours around a NACA0012 airfoil. Viscous-inviscid flow (top) and inviscid flow (bottom). Velocity distribution in $\mathrm{m}^{-1} \mathrm{~s}^{-1}$

Figure 19 depicts the friction coefficient $\left(C_{f}\right)$ as calculated by equation [3.33] at different stages of convergence, compared with the XFOIL values for a NACA0012 airfoil. The friction coefficient is important since this dimensionless parameter relates to the friction drag found in the boundary layer. The smaller the friction drag, the more economical the fuel- 
usage of the aircraft. The $C_{f}$ values converge to the XFOIL solution with an overall error of $4.7 \%$ and a maximum discrepancy of $7.7 \%$ to present an accurate value to use for estimating the overall effects of the drag. Figure 20 shows the velocity that is computed by the auxiliary boundary layer equation [4.3] (in order to avoid the Goldstein singularity) compared with the velocity specified from the inviscid solver. It is compared at the different stages of convergence, showing that the two velocities converge.

Figure 21 shows the unstructured mesh with velocity contours around the NACA0012 airfoil for both the viscous-inviscid and only inviscid flow cases. The viscous-inviscid case also shows the mesh movement depicted by the light grey area around the airfoil. The velocity contours change significantly when the boundary layer solver is coupled to the inviscid flow solver. Figure 22 presents a magnification of the velocity distribution around the leading edge of the airfoil. The coupled solver is very robust when solving the stagnation point present at the leading edge.

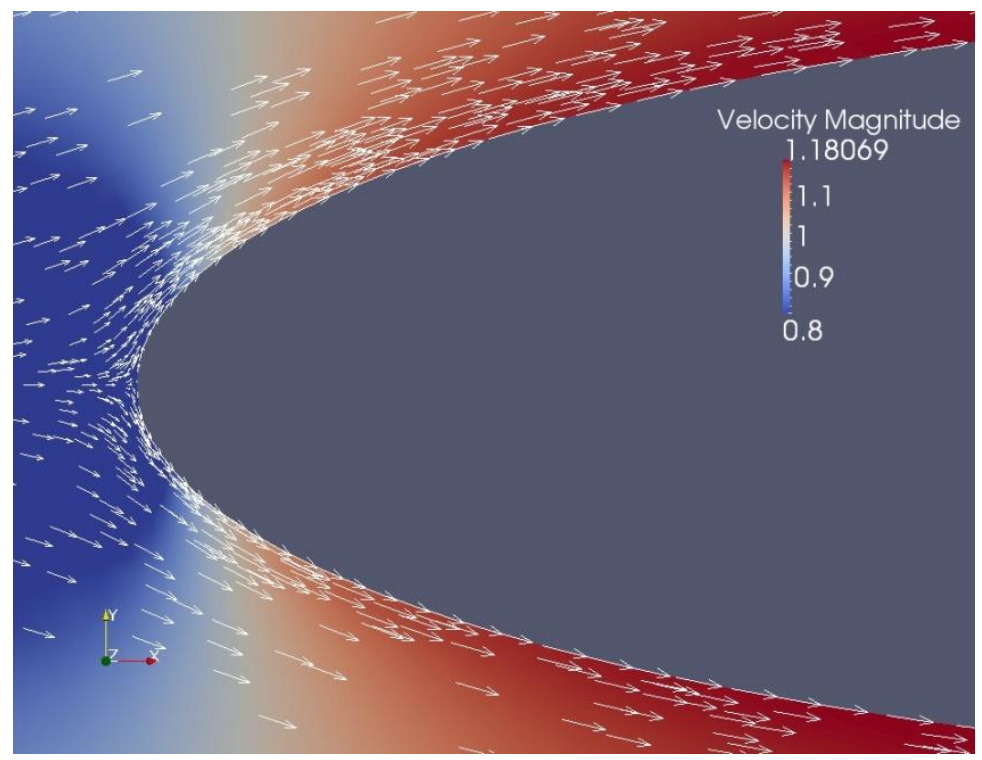

Figure 22: Magnification of the flow across the leading edge of a NACA0012 airfoil. Velocity distribution in $\mathbf{m} . \mathrm{s}^{-1}$

Figure 23 displays the mesh movement around the airfoil compared with the boundary layer thickness predicted by XFOIL 6.9 to an accuracy of 3.95\%. It is evident that as the solver reaches the trailing edge and into the wake, the accuracy decreases. This is due to the assumed wake function (see Section 4.7). 


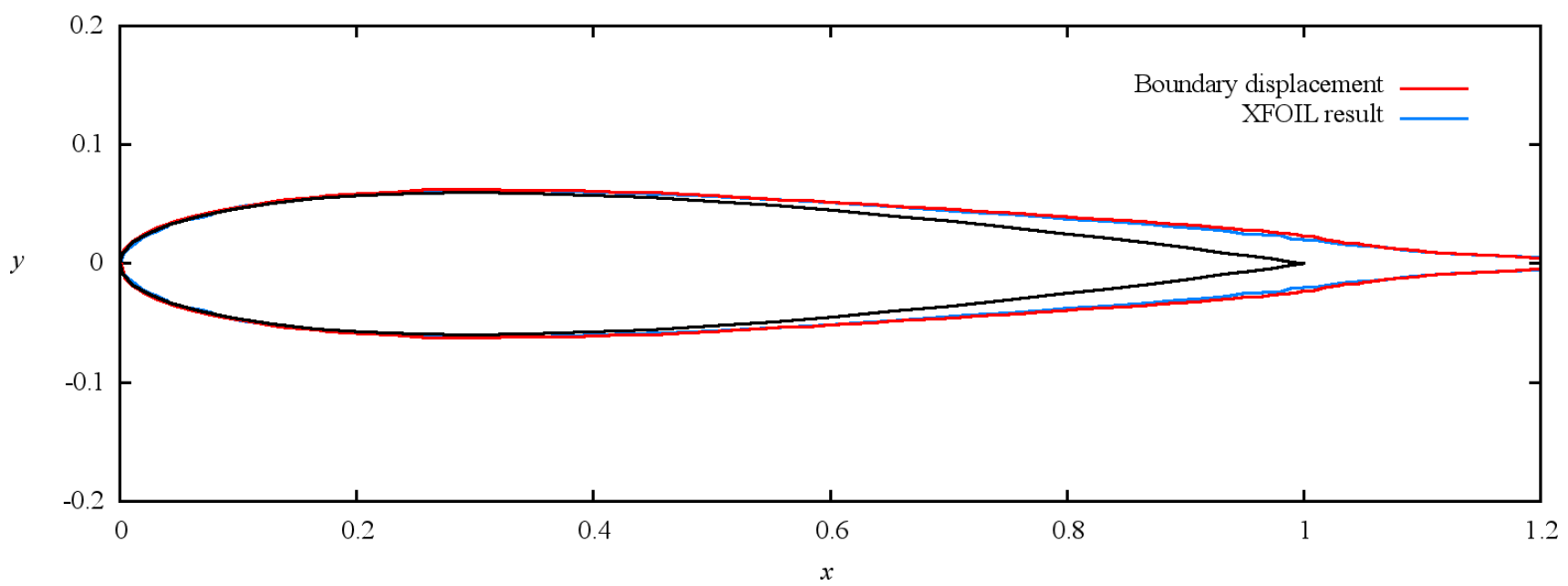

Figure 23: Mesh movement around the NACA0012 airfoil

\subsection{Chapter summary}

In this chapter, the accuracy and robustness of the developed modelling technology were evaluated. For this purpose, two classes of test cases were considered: one with only the boundary layer solver and the other with the viscous-inviscid coupling between the boundary layer solver and an existing inviscid solver. In both cases, the solvers proved to be robust and stable and only tuneable for the sake of convergence by the under-relaxation parameter and the CFL number. The boundary layer cases consisted of flow of a flat plate as well as flow over an inclined plate at different angles of attack. The developed boundary layer modelling scheme was proved second-order accurate. With regards to the second class of test cases, the above problems were again considered, in addition to the flow over a NACA0012 airfoil. Results for the latter were compared with those of the XFOIL program. In all cases, the developed solver was demonstrated to be robust and accurate. 


\section{CHAPTER 6}

\section{CONCLUSIONS}

\subsection{Summary and discussion}

CFD codes are an important tool to visualise and predict flow phenomena. An important engineering aspect of many flow problems is the behaviour of the fluid near a solid boundary. Viscous flow moves from having completely irrotational motion away from the boundary up to the surface of the body where the velocity reaches zero, because of the no-slip condition at the wall. The result is viscous drag. Generally, these effects are solved using the NavierStokes equations. However, to simulate boundary layer flow with most current CFD codes requires extremely fine mesh spacing normal to the wall and is consequently computationally very expensive. The boundary layer is not only important to determine appropriate shapes to minimise drag across a body and thereby save fuel costs or to avoid separation but also to simulate flow through blade cascades in compressors and turbines.

In the light of the above, for the purpose of this work, the flow domain is effectively divided into two regions: the outer (inviscid) flow region and the boundary layer (viscous) region at the surface. A novel viscous-inviscid modelling technology was developed, whereby the viscous region was described by boundary layer equations while the outer region was solved via an incompressible flow solver.

In the case of the viscous boundary layer region, the two-integral method of Drela (1985),was used, obtaining the momentum integral equation in terms of momentum and displacement thickness, to solve flow in the boundary layer and predict the displacement thickness. Drela's 
method is able to solve limited cases of separation and forms the basis of most boundary layer methods used by other researchers such as Wolles and Hoeijmakers (1998), Sekar and Laschka (2005) and Riziotis and Voutsinas (2008). The boundary layer equations were then discretised using the Crank-Nicolson differencing scheme (second-order implicit scheme) to ensure stability. The Crank-Nicolson scheme is unconditionally stable and is an implicit difference method, which has the advantage that the spacing of the grid lines is not limited by stability constraints. These equations were solved point by point as an initial value problem by a local Newton method, since this method is relatively fast to converge, if the initial approximation is close to the solution.

The laminar closure equations reach a singularity at the point where $H_{k}$ reaches 4 , which is referred to as the 'Goldstein singularity' at a boundary layer separation point. One way, in which the problem can be avoided is by solving the inviscid flow and boundary layer simultaneously, however, this is computationally expensive. In this work, the problem was eliminated by allowing the boundary layer to modify the inviscid flow solver it was interacting with by modifying $U_{e}$ via the displacement thickness. The process creates a negative feedback effect, which eliminates the singularity. This is achieved by assuming that the boundary layer is growing on the wall of a two-dimensional channel and solving for the velocity by using an estimated displacement thickness that is updated continuously until the percentage change between the previous and latest displacement thickness falls below a threshold value (here taken as $0.2 \%$ ).

For the purpose of viscous-inviscid coupling, a new and novel method is proposed in this work. It is designed to be both robust and computationally as cost-effective as the quasisimultaneous method, without requiring the selection of an interactive law. It is also advantageous since prior information about the displacement thickness is not needed and the complete flow field can be solved without solving a monolithic system. This is achieved by moving the inviscid mesh at each iteration to reflect the boundary layer displacement. The solution procedure is as follows: the flow solver converges to a solution where the residual is less than the specified tolerance. The solver then uses the boundary layer thickness obtained from the boundary layer solution to move the mesh to the outer edge of the boundary where a slip boundary condition is imposed. The mesh is re-preprocessed and the flow is calculated again until the residual is less than the convergence tolerance after the movement of the mesh. 
The developed modelling technology is thoroughly validated in terms of accuracy and robustness via application to a number of test cases. Two classes of test cases were considered: one with only the boundary layer solver and the other with the viscous-inviscid coupling between the boundary layer solver and an existing inviscid solver. The first class of boundary layer cases consisted of flow of a flat plate as well as flow over an inclined plate at different angles of attack. The second class of test cases involved the same problems, in addition to the flow over a NACA0012 airfoil (results for the latter were compared with those of the XFOIL program). The developed boundary layer modelling scheme was proved second-order accurate. In both cases, the solvers proved to be robust and stable and only tuneable for the sake of convergence by the under-relaxation parameter and the CFL number.

\subsection{Future suggestions}

The methodology developed can be extended in a straightforward manner to treat practical airfoil design problems with transition to turbulent flows and even mild flow separation. The following recommendations for further work are made to expand the range of problems that the interaction method can solve and increase accuracy:

- Expand the two-integral method to solve flow in the turbulent regime by adding the additional parameters and equations given in the work of Drela (1985).

- Expand the two-integral method to solve the transition regime, by adding an amplification variable to the laminar viscous equation.

- Solve the wake with the proper closure equations suggested by Drela (1985) to obtain a more accurate solution for the boundary layer displacement past the trailing edge.

- The inviscid solver is a separate module in this study, which has the potential to be extended to compressible flow as well. Extending to a compressible outer flow solver would allow transonic flow regimes to be modeled as well. This differs from Drela's method (1985) which simultaneously solves the viscous flow linked with a more limited potential flow model. 


\section{REFERENCES}

Anderson, D.A. 2007. Fundamentals of aerodynamics. 4th ed. New York: McGraw-Hill.

Brown, S.N. \& Stewartson, K. 1969. Laminar separation. Annual Review of Fluid Mechanics, $1: 45-72$

Burden, R.L. \& Faires, J.D. 2005. Numerical analysis. 8th ed. Belmont: Thompson Brooks/Cole.

Catherall, D. \& Mangler, K.W. 1966. The integration of the two-dimensional laminar boundary layer equations past the point of vanishing skin friction. Journal of Fluid Mechanics 26:163 - 182 .

Cebeci, T. 1999. An engineering approach to the calculation of aerodynamic flows. Los Angeles, CA: Horizon.

Cebeci, T. \& Cousteix, J. 2005. Modelling and computation of boundary layer flows. Los Angeles, CA: Horizon.

Coenen, E.G.M. 2001. Viscous-inviscid interaction with the quasi-simultaneous method for $2 D$ and $3 D$ airfoil flow. $\mathrm{PhD}$ thesis. Groningen: University of Groningen.

Drela, M. 1985. Two-dimensional transonic aerodynamic design and analysis using the Euler equations. PhD thesis. Cambridge, MA: Department of Aeronautics and Astronautics. Massachusettes Institute of Technology.

Drela M. 2010. Private communication.

Drela, M. \& Giles, M.B. 1987a. Two-dimensional transonic aerodynamics design method. American Institute of Aeronautics and Astronautics Journal, 25(9):1199 - 1206.

Drela, M. \& Giles, M.B. 1987b. Viscous-Inviscid analysis of transonic and low Reynolds number airfoils. American Institute of Aeronautics and Astronautics Journal, 25(10):347 - 1355 .

Drela, M. \& Youngren, H. 2001. XFOIL 6.9 User Primer. Cambridge, MA: Department of Aeronautics and Astronautics. Massachusettes Institute of Technology. 
Falkner, V.M. \& Skan, S.W. 1931. Solution of the boundary layer equations. Philosophical Magazine and Journal of Science, Series 7, 12(80):865 - 897.

Florea, R., Hall, K.C. \& Cizmas, P.G.A. 1998. Reduced-order modelling of unsteady viscous flow in a compressor cascade. American Institute of Aeronautics and Astronautics Journal, 36(6):1039 - 1048.

Gleyzes, C., Cousteix, J. \& Bonnet, J.L. 1985. Theoretical and experimental study of low Reynolds number transitional separation bubbles. Conference on Low Reynolds Number Airfoil Aerodynamics. United States. (June):137-152.

Goldstein, S. 1947. On laminar boundary layer flow near a position of separation. Journal of Mechanics and Applied Mathematics, 1(1):43 - 69.

Hafez, M.M., Habashi, W.G. \& Przybytkowski, S.M. 1991. Transonic viscous-inviscid interaction by a finite element method. International Journal of Numerical Methods in Fluids, 13:309-319.

Hirsch, C. 1995. Numerical computation of internal and external flows. Volume 2: Computational methods for inviscid and viscous flows. Wiltshire:John Wiley.

Hirsch, C. 2001. Numerical computation of internal and external flows. Volume 1: Fundamentals of numerical discretisation. Eastbourne:John Wiley.

Jie, L. \& Zhou, Z. 2007. A structured mesh Euler and interactive boundary layer method for wing/body configurations. Chinese Journal of Aeronautics, 21:19 - 27.

Katz, J. \& Plotkin, A. 2001. Low speed aerodynamic. 2nd ed. New York:Cambridge University Press.

Keller, H.B. 1978. Numerical methods in boundary layer theory. Annual Review of Fluid Mechanics, 10:417-433.

Lagree, P.Y. 2009. Interactive boundary layer or inviscid-viscous interaction. Paris:Institut Jean Le Rond d'Alembert.

Le Balleur, J.C. 1983. Numerical viscous-inviscid interaction in steady and unsteady flows. Proceedings of the Second Symposium, Numerical and Physical Aspects of Aerodynamic Flow, 259 - 284. 
Lock, R. \& Williams, B. 1987. Viscous-inviscid interactions in external aerodynamics. Progress Aerospace Science, 24:51 - 171.

Lighthill, M.J. 1958. On displacement thickness. Journal of Fluid Mechanics, 4:383 - 392.

Malan, A.G. \& Lewis, R.W. 2011. An artificial compressibility CBS method for modelling heat transfer and fluid flow in heterogeneous porous materials. International Journal for Numerical Methods in Engineering, 86(7), In press.

Malan, A.G., Lewis, R.W. \& Nithiarasu, P. 2002. An improved unsteady, artificial compressibility, finite volume scheme for viscous incompressible flows: Part I. Theory and Implementation. International Journal for Numerical Methods in Engineering, 54(5):695 - 714.

Messiter, A.F. 1970. Boundary layer flow near the trailing edge of a flat plate. Journal of Applied Mathematics, 18(1):241 - 257.

Nithiarasu, P. 2003. An efficient artificial compressibility (AC) scheme based on the characteristics based split (CBS) method for incompressible flows. International Journal for Numerical Methods in Engineering, 56:1815 - 1845.

Riziotis, V.A. \& Voutsinas, S.G. 2008. Dynamic stall modelling on airfoils based on strong viscous-inviscid interaction coupling. International Journal of Numerical Methods in Fluids, 56:185 - 208.

Roache, R.J. 1997. Quantification of uncertainty in computational fluid dynamics. Annual Review of Fluid Mechanics, 29:123 - 160.

Schlichting, H. \& Gersten, K. 2000. Boundary layer theory 8th ed. Berlin:Springer-Verlag.

Sekar, W.K. \& Laschka, B. 2005. Calculation of the transonic dip of airfoils using viscousinviscid aerodynamic interaction method. Aerospace Science and Technology, 9:661 671.

White, F.M. 2006. Viscous fluid flow. 3rd ed. Singapore:McGraw-Hill.

Whitfield, D.L. 1978. Analytical description of the complete turbulent boundary layer velocity profile. American Institute of Aeronautics and Astronautics Papers 78-1158. 11th Fluid and Plasma Dynamics Conference. (July):12. 
Soize, C. 1992. Strong coupling between inviscid fluid and boundary layer for airfoils with a sharp edge. II. 2D unsteady case for isolated airfoil and straight blade cascade. La Recherche Aerospatiale. 3:23 -53.

Stewartson, K. 1968. On the flow near the trailing edge of a flat plate II. Proceedings of the Royal Society of London. Series A, Mathematical and Physical Sciences, 306(1486):275 - 290.

Stewartson, K. 1974. Multi-structured boundary layers on flat plates and related bodies. Advances in Applied Mechanics, 14:145 - 293.

Szmelter, J. 2001. Multipoint Aerodynamic wing optimisation in viscous flow. Journal of Aircraft, 38(5):860 - 867.

Tannehill, J.C., Anderson, D.A. \& Pletcher, H.P. 1997. Computational fluid mechanics and heat transfer 2nd ed. New York:Taylor and Francis.

Tani, I. 1977. History of boundary layer theory. Annual Reviews of Fluid Mechanics, 9:87 111.

Veldman, A.E.P. 1981. New, quasi-simultaneous method to calculate interacting boundary layers. American Institute of Aeronautics and Astronautics Journal, 19:79 - 85.

Veldman, A.E.P. 2009. A simple interaction law for viscous-inviscid interaction. Journal of Engineering Mathematics, 65:367 - 383.

Versteeg, H.K. \& Malalasekera, W. 2007. An introduction to computational fluid dynamics: The finite volume method. 2nd ed. Essex:Pearson Education.

White, F.M. 2006. Viscous fluid flow. 3rd ed. Singapore:McGraw-Hill.

Whitfield, D.L. 1978. Analytical description of the complete turbulent boundary layer velocity profile. American Institute of Aeronautics and Astronautics Papers 78-1158. 11th Fluid and Plasma Dynamics Conference. (July):12.

Wolles, B.A. \& Hoeijmakers, H.W.M. 1998. On viscid-inviscid interaction modelling for airfoils as a numerical technique. American Institute of Aeronautics and Astronautics Papers 98-0100. Netherlands:University of Twente. 